\title{
The Weyl product on quasi-Banach modulation spaces
}

\author{
Yuanyuan Chen*, Joachim Toft ${ }^{\dagger}$ and Patrik Wahlberg ${ }^{\ddagger}$ \\ Department of Mathematics \\ Linncus University, 35195 Växjö, Sweden \\ *yuanyuanchen0822@gmail.com \\ ${ }_{j}$ joachim.toft@lnu.se \\ ${ }^{\ddagger}$ patrik.wahlberg@lnu.se
}

Received 8 September 2017

Accepted 12 January 2018

Published 19 June 2019

Communicated by Ari Laptev

\begin{abstract}
We study the bilinear Weyl product acting on quasi-Banach modulation spaces. We find sufficient conditions for continuity of the Weyl product and we derive necessary conditions. The results extend known results for Banach modulation spaces.

Keywords: Pseudodifferential calculus; Weyl product; modulation spaces; quasi-Banach spaces; Gabor frames.

Mathematics Subject Classification: 35S05, 47G30, 42B35, 46A16, 46E35, 46F05
\end{abstract}

\section{Introduction}

In this paper, we study the Weyl product acting on weighted modulation spaces with Lebesgue parameters in $(0, \infty]$. We work out conditions on the weights and the Lebesgue parameters that are sufficient for continuity of the Weyl product, and we also prove necessary conditions.

The Weyl product or twisted product is the product of symbols in the Weyl calculus of pseudodifferential operators corresponding to operator composition. This means that the Weyl product

$$
\left(a_{1}, a_{2}\right) \mapsto a_{1} \# a_{2},
$$

‡Corresponding author.

This is an Open Access article published by World Scientific Publishing Company. It is distributed under the terms of the Creative Commons Attribution 4.0 (CC-BY) License. Further distribution of this work is permitted, provided the original work is properly cited. 
of two distributions $a_{1}$ and $a_{2}$ defined on the phase space $T^{*} \mathbf{R}^{d} \simeq \mathbf{R}^{2 d}$ is defined by

$$
\mathrm{Op}^{w}\left(a_{1} \# a_{2}\right)=\mathrm{Op}^{w}\left(a_{1}\right) \circ \mathrm{Op}^{w}\left(a_{2}\right),
$$

provided the composition is well-defined.

Our result on sufficient conditions is as follows. Suppose $\omega_{j}, j=0,1,2$, are moderate weights on $\mathbf{R}^{4 d}$ that satisfy

$$
\omega_{0}(Z+X, Z-X) \lesssim \omega_{1}(Y+X, Y-X) \omega_{2}(Z+Y, Z-Y), \quad X, Y, Z \in \mathbf{R}^{2 d} .
$$

Suppose $p_{j}, q_{j} \in(0, \infty], j=0,1,2$, satisfy

$$
\frac{1}{p_{0}} \leq \frac{1}{p_{1}}+\frac{1}{p_{2}}
$$

and either

$$
q_{1}, q_{2} \leq q_{0} \leq \min \left(1, p_{0}\right)
$$

or

$$
\min \left(1, p_{0}\right) \leq q_{1}, q_{2} \leq q_{0} \quad \text { and } \quad \frac{1}{\min \left(1, p_{0}\right)}+\frac{1}{q_{0}} \leq \frac{1}{q_{1}}+\frac{1}{q_{2}}
$$

Denote the Gelfand-Shilov space of order $\frac{1}{2}$ by $\mathcal{S}_{1 / 2}$, and the weighted modulation space with Lebesgue parameters $p, q>0$ and with weight $\omega$ by $\mathcal{M}_{(\omega)}^{p, q}$. Then the map $\left(a_{1}, a_{2}\right) \mapsto a_{1} \# a_{2}$ from $\mathcal{S}_{1 / 2}\left(\mathbf{R}^{2 d}\right) \times \mathcal{S}_{1 / 2}\left(\mathbf{R}^{2 d}\right)$ to $\mathcal{S}_{1 / 2}\left(\mathbf{R}^{2 d}\right)$ extends uniquely to a continuous map from $\mathcal{M}_{\left(\omega_{1}\right)}^{p_{1}, q_{1}}\left(\mathbf{R}^{2 d}\right) \times \mathcal{M}_{\left(\omega_{2}\right)}^{p_{2}, q_{2}}\left(\mathbf{R}^{2 d}\right)$ to $\mathcal{M}_{\left(\omega_{0}\right)}^{p_{0}, q_{0}}\left(\mathbf{R}^{2 d}\right)$, and

$$
\left\|a_{1} \# a_{2}\right\|_{\mathcal{M}_{\left(\omega_{0}\right)}^{p_{0}, q_{0}}} \lesssim\left\|a_{1}\right\|_{\mathcal{M}_{\left(\omega_{1}\right)}^{p_{1}, q_{1}}}\left\|a_{2}\right\|_{\mathcal{M}_{\left(\omega_{2}\right)}^{p_{2}, q_{2}}} .
$$

As a consequence for unweighted modulation spaces, we obtain new conditions on Lebesgue parameters that are sufficient for $\mathcal{M}^{p, q}\left(\mathbf{R}^{2 d}\right)$ to be an algebra: $p, q \in$ $(0, \infty]$ and $q \leq \min (1, p)$.

The necessary conditions we deduce are as follows. Suppose (1.1) holds for all $a_{1}, a_{2} \in \mathscr{S}\left(\mathbf{R}^{2 d}\right)$, for a triple of polynomial type weights $\omega_{j}, j=0,1,2$ interrelated in a certain way, see (3.7). Then

$$
\frac{1}{p_{0}} \leq \frac{1}{p_{1}}+\frac{1}{p_{2}}, \quad \frac{1}{p_{0}} \leq \frac{1}{q_{1}}+\frac{1}{q_{2}} \quad \text { and } \quad q_{1}, q_{2} \leq q_{0},
$$

which are strictly weaker than the sufficient conditions.

Our results for the Weyl product are special cases of results formulated and proved for a family of pseudodifferential calculi parametrized by real matrices $A \in$ $\mathbf{R}^{d \times d}$. In fact, we work with a symbol product indexed by $A \in \mathbf{R}^{d \times d}$, denoted and defined by

$$
\mathrm{Op}_{A}\left(a \# \#_{A} b\right)=\mathrm{Op}_{A}(a) \circ \mathrm{Op}_{A}(b),
$$

where $\mathrm{Op}_{A}(a)$ is the $A$-indexed pseudodifferential operator with symbol $a$. This family of calculi contains the Weyl quantization as the special case $A=\frac{1}{2} I$.

The sufficient conditions and the necessary conditions that we find extend results [7. 23. where the same problem was studied for the narrower range of Lebesgue 
parameters $[1, \infty]$. In the latter case, modulation spaces are Banach spaces, whereas they are merely quasi-Banach spaces if a Lebesgue parameter is smaller than one.

The Weyl product on Banach modulation spaces has been studied in e.g. 7, 18, 20, 23, 26, 29, 30. In 7] conditions on the Lebesgue parameters were found that are both necessary and sufficient for continuity of the Weyl product, thus characterizing the Weyl product acting on Banach modulation spaces.

One possible reason that we do not obtain characterizations in the full range of Lebesgue parameters $(0, \infty]$ is that new difficulties arise as soon as a Lebesgue parameter is smaller than one. The available techniques are quite different, and many tools that are useful in the Banach space case, e.g. duality and complex interpolation, are not applicable or fraught with subtle difficulties.

Our technique to prove the sufficient conditions consists of a discretization of the Weyl product by means of a Gabor frame. This reduces the continuity of the Weyl product to the continuity of certain infinite-dimensional matrix operators. A similar idea has been developed in 38.

The paper is organized as follows. Section 2 fixes notation and gives the background on Gelfand-Shilov function and distribution spaces, pseudo-differential calculi, modulation spaces, Gabor frames, and symbol product results for Banach modulation spaces.

Section 3 contains the result on sufficient conditions for continuity on quasiBanach modulation spaces (Theorem 3.1). Section 4 contains the result on necessary conditions for continuity on quasi-Banach modulation spaces (Theorem 4.3). Finally in Appendix, we show a Fubini type result for Gelfand-Shilov distributions that is needed in the definition of the short-time Fourier transform (STFT) of a GelfandShilov distribution.

\section{Preliminaries}

\subsection{Weight functions}

A weight on $\mathbf{R}^{d}$ is a positive function $\omega \in L_{\mathrm{loc}}^{\infty}\left(\mathbf{R}^{d}\right)$ such that $1 / \omega \in L_{\mathrm{loc}}^{\infty}\left(\mathbf{R}^{d}\right)$. We usually assume that $\omega$ is $(v-)$ moderate, for some positive function $v \in L_{\mathrm{loc}}^{\infty}\left(\mathbf{R}^{d}\right)$. This means

$$
\omega(x+y) \lesssim \omega(x) v(y), \quad x, y \in \mathbf{R}^{d} .
$$

Here, $f(\theta) \lesssim g(\theta)$ means that $f(\theta) \leq c g(\theta)$ holds uniformly for all $\theta$ in the intersection of the domains of $f$ and $g$ for some constant $c>0$, and we write $f \asymp g$ when $f \lesssim g \lesssim f$. Note that (2.1) implies the estimates

$$
v(-x)^{-1} \lesssim \omega(x) \lesssim v(x), \quad x \in \mathbf{R}^{d} .
$$

If $v$ in (2.1) can be chosen as a polynomial, then $\omega$ is called polynomially moderate or a weight of polynomial type. We let $\mathscr{P}\left(\mathbf{R}^{d}\right)$ and $\mathscr{P}_{E}\left(\mathbf{R}^{d}\right)$ be the sets of all weights of polynomial type and moderate weights on $\mathbf{R}^{d}$, respectively. 
If $\omega \in \mathscr{P}_{E}\left(\mathbf{R}^{d}\right)$, then there exists $r>0$ such that $\omega$ is $v$-moderate for $v(x)=e^{r|x|}$ [19]. Hence by (2.2), for any $\omega \in \mathscr{P}_{E}\left(\mathbf{R}^{d}\right)$, there is $r>0$ such that

$$
e^{-r|x|} \lesssim \omega(x) \lesssim e^{r|x|}, \quad x \in \mathbf{R}^{d} .
$$

A weight $v$ is called submultiplicative if $v$ is even and (2.1) holds with $\omega=v$. In the paper, $v$ and $v_{j}$ for $j \geq 0$ will denote submultiplicative weights if not otherwise stated.

\subsection{Gelfand-Shilov spaces}

Let $h, s \in \mathbf{R}_{+}$be fixed. Then $\mathcal{S}_{s, h}\left(\mathbf{R}^{d}\right)$ is the set of all $f \in C^{\infty}\left(\mathbf{R}^{d}\right)$ such that

$$
\|f\|_{\mathcal{S}_{s, h}} \equiv \sup \frac{\left|x^{\beta} \partial^{\alpha} f(x)\right|}{h^{|\alpha+\beta|}(\alpha ! \beta !)^{s}}
$$

is finite, where the supremum is taken over all $\alpha, \beta \in \mathbf{N}^{d}$ and $x \in \mathbf{R}^{d}$.

Obviously $\mathcal{S}_{s, h}$ is a Banach space which increases with $h$ and $s$, and it is contained in the Schwartz space $\mathscr{S}$. (Inclusions of function and distribution spaces understand embeddings.) The topological dual $\mathcal{S}_{s, h}^{\prime}\left(\mathbf{R}^{d}\right)$ of $\mathcal{S}_{s, h}\left(\mathbf{R}^{d}\right)$ is a Banach space which contains $\mathscr{S}^{\prime}\left(\mathbf{R}^{d}\right)$ (the tempered distributions). If $s>\frac{1}{2}$, then $\mathcal{S}_{s, h}$ and $\bigcup_{h>0} \mathcal{S}_{1 / 2, h}$ contain all finite linear combinations of Hermite functions.

The (Fourier invariant) Gelfand-Shilov spaces $\mathcal{S}_{s}\left(\mathbf{R}^{d}\right)$ and $\Sigma_{s}\left(\mathbf{R}^{d}\right)$ are the inductive and projective limits respectively of $\mathcal{S}_{s, h}\left(\mathbf{R}^{d}\right)$ with respect to $h$. This implies

$$
\mathcal{S}_{s}\left(\mathbf{R}^{d}\right)=\bigcup_{h>0} \mathcal{S}_{s, h}\left(\mathbf{R}^{d}\right) \quad \text { and } \quad \Sigma_{s}\left(\mathbf{R}^{d}\right)=\bigcap_{h>0} \mathcal{S}_{s, h}\left(\mathbf{R}^{d}\right) .
$$

The topology for $\mathcal{S}_{s}\left(\mathbf{R}^{d}\right)$ is the strongest topology such that each inclusion $\mathcal{S}_{s, h}\left(\mathbf{R}^{d}\right) \subseteq \mathcal{S}_{s}\left(\mathbf{R}^{d}\right)$ is continuous. The projective limit $\Sigma_{s}\left(\mathbf{R}^{d}\right)$ is a Fréchet space with seminorms $\|\cdot\|_{\mathcal{S}_{s, h}}, h>0$. It holds $\mathcal{S}_{s}\left(\mathbf{R}^{d}\right) \neq\{0\}$ if and only if $s \geq \frac{1}{2}$, and $\Sigma_{s}\left(\mathbf{R}^{d}\right) \neq\{0\}$ if and only if $s>\frac{1}{2}$.

For every $\varepsilon>0$ and $s>0$,

$$
\Sigma_{s}\left(\mathbf{R}^{d}\right) \subseteq \mathcal{S}_{s}\left(\mathbf{R}^{d}\right) \subseteq \Sigma_{s+\varepsilon}\left(\mathbf{R}^{d}\right) .
$$

The Gelfand-Shilov distribution spaces $\mathcal{S}_{s}^{\prime}\left(\mathbf{R}^{d}\right)$ and $\Sigma_{s}^{\prime}\left(\mathbf{R}^{d}\right)$ are the projective and inductive limits respectively of $\mathcal{S}_{s, h}^{\prime}\left(\mathbf{R}^{d}\right)$. Hence if $s \geq \frac{1}{2}$ and $t>\frac{1}{2}$ then

$$
\mathcal{S}_{s}^{\prime}\left(\mathbf{R}^{d}\right)=\bigcap_{h>0} \mathcal{S}_{s, h}^{\prime}\left(\mathbf{R}^{d}\right) \quad \text { and } \quad \Sigma_{t}^{\prime}\left(\mathbf{R}^{d}\right)=\bigcup_{h>0} \mathcal{S}_{t, h}^{\prime}\left(\mathbf{R}^{d}\right)
$$

The space $\mathcal{S}_{s}^{\prime}\left(\mathbf{R}^{d}\right)$ is the topological dual of $\mathcal{S}_{s}\left(\mathbf{R}^{d}\right)$, and if $s>\frac{1}{2}$ then $\Sigma_{s}^{\prime}\left(\mathbf{R}^{d}\right)$ is the topological dual of $\Sigma_{s}\left(\mathbf{R}^{d}\right)[12]$.

The action of a distribution $f$ on a test function $\phi$ is written $\langle f, \phi\rangle$, and the conjugate linear action is written $(u, \phi)=\langle u, \bar{\phi}\rangle$, consistent with the $L^{2}$ inner product $(\cdot, \cdot)=(\cdot, \cdot)_{L^{2}}$ which is conjugate linear in the second argument. 
The Gelfand-Shilov (distribution) spaces enjoy many invariance properties, for instance under translation, dilation, tensorization, coordinate transformations and (partial) Fourier transformation.

We use the normalization

$$
\mathscr{F} f(\xi)=\widehat{f}(\xi)=(2 \pi)^{-\frac{d}{2}} \int_{\mathbf{R}^{d}} f(x) e^{-i\langle x, \xi\rangle} d x, \quad \xi \in \mathbf{R}^{d},
$$

of the Fourier transform of $f \in L^{1}\left(\mathbf{R}^{d}\right)$, where $\langle\cdot, \cdot\rangle$ denotes the scalar product on $\mathbf{R}^{d}$. The Fourier transform $\mathscr{F}$ extends uniquely to homeomorphisms on $\mathscr{S}^{\prime}\left(\mathbf{R}^{d}\right)$, $\mathcal{S}_{s}^{\prime}\left(\mathbf{R}^{d}\right)$ and $\Sigma_{s}^{\prime}\left(\mathbf{R}^{d}\right)$, and restricts to homeomorphisms on $\mathscr{S}\left(\mathbf{R}^{d}\right), \mathcal{S}_{s}\left(\mathbf{R}^{d}\right)$ and $\Sigma_{s}\left(\mathbf{R}^{d}\right)$, and to a unitary operator on $L^{2}\left(\mathbf{R}^{d}\right)$.

The symplectic Fourier transform of $a \in \mathcal{S}_{s}\left(\mathbf{R}^{2 d}\right)$ where $s \geq \frac{1}{2}$ is defined by

$$
\mathscr{F}_{\sigma} a(X)=\pi^{-d} \int_{\mathbf{R}^{2 d}} a(Y) e^{2 i \sigma(X, Y)} d Y,
$$

where $\sigma$ is the symplectic form

$$
\sigma(X, Y)=\langle y, \xi\rangle-\langle x, \eta\rangle, \quad X=(x, \xi) \in \mathbf{R}^{2 d}, \quad Y=(y, \eta) \in \mathbf{R}^{2 d} .
$$

Since $\mathscr{F}_{\sigma} a(x, \xi)=2^{d} \mathscr{F} a(-2 \xi, 2 x)$, the definition of $\mathscr{F}_{\sigma}$ extends in the same way as $\mathscr{F}$.

Let $\phi \in \mathcal{S}_{s}\left(\mathbf{R}^{d}\right) \backslash\{0\}$. The short-time Fourier transform (STFT) $V_{\phi} f$ of $f \in$ $\mathcal{S}_{s}^{\prime}\left(\mathbf{R}^{d}\right)$ is the distribution on $\mathbf{R}^{2 d}$ defined by

$$
V_{\phi} f(x, \xi)=\mathscr{F}(f \overline{\phi(\cdot-x)})(\xi)=(2 \pi)^{-\frac{d}{2}}\left(f, \phi(\cdot-x) e^{i\langle\cdot, \xi\rangle}\right) .
$$

Note that $f \overline{\phi(\cdot-x)} \in \mathcal{S}_{s}^{\prime}\left(\mathbf{R}^{d}\right)$ for fixed $x \in \mathbf{R}^{d}$, and therefore its Fourier transform is an element in $\mathcal{S}_{s}^{\prime}\left(\mathbf{R}^{d}\right)$. The fact that the Fourier transform is actually a smooth function given by the formula (2.5) is proved in Appendix.

If $T(f, \phi) \equiv V_{\phi} f$ for $f, \phi \in \mathcal{S}_{1 / 2}\left(\mathbf{R}^{d}\right)$, then $T$ extends uniquely to sequentially continuous mappings

$$
\begin{aligned}
& T: \mathcal{S}_{s}^{\prime}\left(\mathbf{R}^{d}\right) \times \mathcal{S}_{s}\left(\mathbf{R}^{d}\right) \rightarrow \mathcal{S}_{s}^{\prime}\left(\mathbf{R}^{2 d}\right) \cap C^{\infty}\left(\mathbf{R}^{2 d}\right), \\
& T: \mathcal{S}_{s}^{\prime}\left(\mathbf{R}^{d}\right) \times \mathcal{S}_{s}^{\prime}\left(\mathbf{R}^{d}\right) \rightarrow \mathcal{S}_{s}^{\prime}\left(\mathbf{R}^{2 d}\right),
\end{aligned}
$$

and similarly when $\mathcal{S}_{s}$ and $\mathcal{S}_{s}^{\prime}$ are replaced by $\Sigma_{s}$ and $\Sigma_{s}^{\prime}$, respectively, or by $\mathscr{S}$ and $\mathscr{S}^{\prime}$, respectively [6, 33].

Similar properties hold true if instead $T(f, \phi)=W_{f, \phi}$, where $W_{f, \phi}$ is the crossWigner distribution of $f \in \mathcal{S}_{s}^{\prime}\left(\mathbf{R}^{d}\right)$ and $\phi \in \mathcal{S}_{s}\left(\mathbf{R}^{d}\right)$, given by

$$
W_{f, \phi}(x, \xi) \equiv \mathscr{F}(f(x+\cdot / 2) \overline{\phi(x-\cdot / 2)})(\xi) .
$$

If $q \in[1, \infty], \omega \in \mathscr{P}_{E}\left(\mathbf{R}^{d}\right), f \in L_{(\omega)}^{q}\left(\mathbf{R}^{d}\right)$ and $\phi \in \Sigma_{1}\left(\mathbf{R}^{d}\right)$ then $V_{\phi} f$ and $W_{f, \phi}$ take the forms

$$
V_{\phi} f(x, \xi)=(2 \pi)^{-\frac{d}{2}} \int_{\mathbf{R}^{d}} f(y) \overline{\phi(y-x)} e^{-i\langle y, \xi\rangle} d y
$$


and

$$
W_{f, \phi}(x, \xi)=(2 \pi)^{-\frac{d}{2}} \int_{\mathbf{R}^{d}} f(x+y / 2) \overline{\phi(x-y / 2)} e^{-i\langle y, \xi\rangle} d y .
$$

Here, $L_{(\omega)}^{p}\left(\mathbf{R}^{d}\right)$ for $p \in(0, \infty]$ and $\omega \in \mathscr{P}_{E}\left(\mathbf{R}^{d}\right)$ denotes the space of all $f \in$ $L_{\text {loc }}^{p}\left(\mathbf{R}^{d}\right)$ such that $f \omega \in L^{p}\left(\mathbf{R}^{d}\right)$, and $\|f\|_{L_{(\omega)}^{p}}=\|f \omega\|_{L^{p}}$.

For $a \in \mathcal{S}_{1 / 2}^{\prime}\left(\mathbf{R}^{2 d}\right)$ and $\Phi \in \mathcal{S}_{1 / 2}\left(\mathbf{R}^{2 d}\right) \backslash 0$ the symplectic STFT $\mathcal{V}_{\Phi} a$ of $a$ with respect to $\Phi$ is defined similarly as the STFT by

$$
\mathcal{V}_{\Phi} a(X, Y)=\mathscr{F}_{\sigma}(a \overline{\Phi(\cdot-X)})(Y), \quad X, Y \in \mathbf{R}^{2 d} .
$$

There are several ways to characterize Gelfand-Shilov function and distribution spaces, for example in terms of expansions with respect to Hermite functions [13, 24], or in terms of the Fourier transform and the STFT [5, 21] 33, 37].

\subsection{An extended family of pseudodifferential calculi}

We consider a family of pseudodifferential calculi parameterized by the real $d \times d$ matrices, denoted $\mathbf{M}(d, \mathbf{R})\left[3\right.$, 36. Let $s \geq \frac{1}{2}$, let $a \in \mathcal{S}_{s}\left(\mathbf{R}^{2 d}\right)$ and let $A \in \mathbf{M}(d, \mathbf{R})$ be fixed. The pseudodifferential operator $\mathrm{Op}_{A}(a)$ is the linear and continuous operator

$$
\mathrm{Op}_{A}(a) f(x)=(2 \pi)^{-d} \iint_{\mathbf{R}^{2 d}} a(x-A(x-y), \xi) f(y) e^{i\langle x-y, \xi\rangle} d y d \xi,
$$

when $f \in \mathcal{S}_{s}\left(\mathbf{R}^{d}\right)$. For $a \in \mathcal{S}_{s}^{\prime}\left(\mathbf{R}^{2 d}\right)$ the operator $\mathrm{Op}_{A}(a)$ is defined as the linear and continuous operator from $\mathcal{S}_{s}\left(\mathbf{R}^{d}\right)$ to $\mathcal{S}_{s}^{\prime}\left(\mathbf{R}^{d}\right)$ with distribution kernel

$$
K_{a, A}(x, y)=(2 \pi)^{-\frac{d}{2}} \mathscr{F}_{2}^{-1} a(x-A(x-y), x-y) .
$$

Here $\mathscr{F}_{2} F$ is the partial Fourier transform of $F(x, y) \in \mathcal{S}_{s}^{\prime}\left(\mathbf{R}^{2 d}\right)$ with respect to the $y$ variable. This definition makes sense since

$$
\mathscr{F}_{2} \quad \text { and } \quad F(x, y) \mapsto F(x-A(x-y), x-y),
$$

are homeomorphisms on $\mathcal{S}_{s}^{\prime}\left(\mathbf{R}^{2 d}\right)$.

An important special case is $A=t I$, with $t \in \mathbf{R}$ and $I \in \mathbf{M}(d, \mathbf{R})$ denoting the identity matrix. In this case, we write $\mathrm{Op}_{t}(a)=\mathrm{Op}_{t I}(a)$. The normal or KohnNirenberg representation $a(x, D)$ corresponds to $t=0$, and the Weyl quantization $\mathrm{Op}^{w}(a)$ corresponds to $t=\frac{1}{2}$. Thus

$$
a(x, D)=\mathrm{Op}_{0}(a)=\mathrm{Op}(a) \quad \text { and } \quad \mathrm{Op}^{w}(a)=\mathrm{Op}_{1 / 2}(a) .
$$

The Weyl calculus is connected to the Wigner distribution with the formula

$$
\begin{aligned}
\left(\mathrm{Op}^{w}(a) f, g\right)_{L^{2}\left(\mathbf{R}^{d}\right)} & =(2 \pi)^{-\frac{d}{2}}\left(a, W_{g, f}\right)_{L^{2}\left(\mathbf{R}^{2 d}\right)}, \\
a & \in \mathcal{S}_{1 / 2}^{\prime}\left(\mathbf{R}^{2 d}\right), \quad f, g \in \mathcal{S}_{1 / 2}\left(\mathbf{R}^{d}\right) .
\end{aligned}
$$

For every $a_{1} \in \mathcal{S}_{s}^{\prime}\left(\mathbf{R}^{2 d}\right)$ and $A_{1}, A_{2} \in \mathbf{M}(d, \mathbf{R})$, there is a unique $a_{2} \in \mathcal{S}_{s}^{\prime}\left(\mathbf{R}^{2 d}\right)$ such that $\mathrm{Op}_{A_{1}}\left(a_{1}\right)=\mathrm{Op}_{A_{2}}\left(a_{2}\right)$. The following restatement of [36, Proposition 2.1] explains the relations between $a_{1}$ and $a_{2}$. 
Proposition 2.1. Let $a_{1}, a_{2} \in \mathcal{S}_{1 / 2}^{\prime}\left(\mathbf{R}^{2 d}\right)$ and $A_{1}, A_{2} \in \mathbf{M}(d, \mathbf{R})$. Then

$$
\mathrm{Op}_{A_{1}}\left(a_{1}\right)=\mathrm{Op}_{A_{2}}\left(a_{2}\right) \Leftrightarrow e^{i\left\langle A_{2} D_{\xi}, D_{x}\right\rangle} a_{2}(x, \xi)=e^{i\left\langle A_{1} D_{\xi}, D_{x}\right\rangle} a_{1}(x, \xi) .
$$

\subsection{Modulation spaces}

Let $\phi \in \mathcal{S}_{1 / 2}\left(\mathbf{R}^{d}\right) \backslash 0, p, q \in(0, \infty]$ and $\omega \in \mathscr{P}_{E}\left(\mathbf{R}^{2 d}\right)$. The modulation space $M_{(\omega)}^{p, q}\left(\mathbf{R}^{d}\right)$ is the set of all $f \in \mathcal{S}_{1 / 2}^{\prime}\left(\mathbf{R}^{d}\right)$ such that $V_{\phi} f \in L_{(\omega)}^{p, q}\left(\mathbf{R}^{2 d}\right)$, and $M_{(\omega)}^{p, q}\left(\mathbf{R}^{d}\right)$ is equipped with the quasi-norm

$$
f \mapsto\|f\|_{M_{(\omega)}^{p, q}} \equiv\left\|V_{\phi} f\right\|_{L_{(\omega)}^{p, q}}
$$

Here $L_{(\omega)}^{p, q}\left(\mathbf{R}^{2 d}\right)$ is the space of all complex-valued measurable functions $F$ on $\mathbf{R}^{2 d}$ such that $\|F\|_{L_{(\omega)}^{p, q}}<\infty$, where

$$
\|F\|_{L_{(\omega)}^{p, q}} \equiv\left\|F_{p, \omega}\right\|_{L^{q}} \quad \text { with } \quad F_{p, \omega}(\xi) \equiv\|F(\cdot, \xi) \omega(\cdot, \xi)\|_{L^{p}}, \quad \xi \in \mathbf{R}^{d} .
$$

On the even-dimensional phase space $\mathbf{R}^{2 d}$, one may define modulation spaces based on the symplectic STFT. Thus if $\omega \in \mathscr{P}_{E}\left(\mathbf{R}^{4 d}\right), p, q \in(0, \infty]$ and $\Phi \in$ $\mathcal{S}_{1 / 2}\left(\mathbf{R}^{2 d}\right) \backslash 0$ are fixed, the symplectic modulation space $\mathcal{M}_{(\omega)}^{p, q}\left(\mathbf{R}^{2 d}\right)$ is obtained by replacing the STFT $a \mapsto V_{\Phi} a$ by the symplectic STFT $a \mapsto \mathcal{V}_{\Phi} a$ in (2.10). It holds (cf. 7])

$$
\mathcal{M}_{(\omega)}^{p, q}\left(\mathbf{R}^{2 d}\right)=M_{\left(\omega_{0}\right)}^{p, q}\left(\mathbf{R}^{2 d}\right), \quad \omega(x, \xi, y, \eta)=\omega_{0}(x, \xi,-2 \eta, 2 y),
$$

so all properties that are valid for $M_{(\omega)}^{p, q}$ carry over to $\mathcal{M}_{(\omega)}^{p, q}$.

In the following propositions, we list some properties of modulation spaces and refer to 8 11, 17, 32, for proofs.

Proposition 2.2. Let $p, q \in(0, \infty]$.

(1) If $\omega \in \mathscr{P}_{E}\left(\mathbf{R}^{2 d}\right)$ then $\Sigma_{1}\left(\mathbf{R}^{d}\right) \subseteq M_{(\omega)}^{p, q}\left(\mathbf{R}^{d}\right) \subseteq \Sigma_{1}^{\prime}\left(\mathbf{R}^{d}\right)$.

(2) If $\omega \in \mathscr{P}_{E}\left(\mathbf{R}^{2 d}\right)$ satisfies (2.3) for every $r>0$, then $\mathcal{S}_{1}\left(\mathbf{R}^{d}\right) \subseteq M_{(\omega)}^{p, q}\left(\mathbf{R}^{d}\right) \subseteq$ $\mathcal{S}_{1}^{\prime}\left(\mathbf{R}^{d}\right)$.

(3) If $\omega \in \mathscr{P}\left(\mathbf{R}^{2 d}\right)$ then $\mathscr{S}\left(\mathbf{R}^{d}\right) \subseteq M_{(\omega)}^{p, q}\left(\mathbf{R}^{d}\right) \subseteq \mathscr{S}^{\prime}\left(\mathbf{R}^{d}\right)$.

Proposition 2.3. Let $r \in(0,1], p, q, p_{j}, q_{j} \in(0, \infty]$ and $\omega, \omega_{j}, v \in \mathscr{P}_{E}\left(\mathbf{R}^{2 d}\right)$, $j=1,2$, satisfy $r \leq \min (p, q), p_{1} \leq p_{2}, q_{1} \leq q_{2}, \omega_{2} \lesssim \omega_{1}$, and let $\omega$ be $v$-moderate.

(1) If $\phi \in M_{(v)}^{r}\left(\mathbf{R}^{d}\right) \backslash 0$ then $f \in M_{(\omega)}^{p, q}\left(\mathbf{R}^{d}\right)$ if and only if (2.10) is finite. In particular $M_{(\omega)}^{p, q}\left(\mathbf{R}^{d}\right)$ is independent of the choice of $\phi \in M_{(v)}^{r}\left(\mathbf{R}^{d}\right) \backslash 0$. The space $M_{(\omega)}^{p, q}\left(\mathbf{R}^{d}\right)$ is a quasi-Banach space under the quasi-norm (2.10), and different choices of $\phi$ give rise to equivalent quasi-norms. If $p, q \geq 1$ then $M_{(\omega)}^{p, q}\left(\mathbf{R}^{d}\right)$ is a Banach space with norm (2.10).

(2) $M_{\left(\omega_{1}\right)}^{p_{1}, q_{1}}\left(\mathbf{R}^{d}\right) \subseteq M_{\left(\omega_{2}\right)}^{p_{2}, q_{2}}\left(\mathbf{R}^{d}\right)$. 
We will rely heavily on Gabor expansions so we need the following concepts. The operators in Definition 2.4 are well defined and continuous by the analysis in [17, Chaps. 11-14].

Definition 2.4. Let $\Lambda \subseteq \mathbf{R}^{d}$ be a lattice, let $\Lambda^{2}=\Lambda \times \Lambda \subseteq \mathbf{R}^{2 d}$, let $\omega, v \in$ $\mathscr{P}_{E}\left(\mathbf{R}^{2 d}\right)$ be such that $\omega$ is $v$-moderate, and let $\phi, \psi \in M_{(v)}^{1}\left(\mathbf{R}^{d}\right)$.

(1) The Gabor analysis operator $C_{\phi}=C_{\phi}^{\Lambda}$ is the operator from $M_{(\omega)}^{\infty}\left(\mathbf{R}^{d}\right)$ to $\ell_{(\omega)}^{\infty}\left(\Lambda^{2}\right)$ given by

$$
C_{\phi}^{\Lambda} f \equiv\left\{V_{\phi} f(j, \iota)\right\}_{j, \iota \in \Lambda} .
$$

(2) The Gabor synthesis operator $D_{\psi}=D_{\psi}^{\Lambda}$ is the operator from $\ell_{(\omega)}^{\infty}\left(\Lambda^{2}\right)$ to $M_{(\omega)}^{\infty}\left(\mathbf{R}^{d}\right)$ given by

$$
D_{\psi}^{\Lambda} c \equiv \sum_{j, \iota \in \Lambda} c(j, \iota) e^{i\langle\cdot, \iota\rangle} \psi(\cdot-j) .
$$

(3) The Gabor frame operator $S_{\phi, \psi}=S_{\phi, \psi}^{\Lambda}$ is the operator on $M_{(\omega)}^{\infty}\left(\mathbf{R}^{d}\right)$ given by $D_{\psi}^{\Lambda} \circ C_{\phi}^{\Lambda}$, i.e.

$$
S_{\phi, \psi}^{\Lambda} f \equiv \sum_{j, \iota \in \Lambda} V_{\phi} f(j, \iota) e^{i\langle\cdot, \iota\rangle} \psi(\cdot-j) .
$$

The following result is a consequence of [17, Theorem 13.1.1] (see also [16, Theorem S]).

Proposition 2.5. Suppose $v \in \mathscr{P}_{E}\left(\mathbf{R}^{2 d}\right)$ is submultiplicative, and let $\phi \in$ $M_{(v)}^{1}\left(\mathbf{R}^{d}\right) \backslash 0$. There is a constant $\theta_{0}>0$ such that the Gabor frame operator $S_{\phi, \phi}^{\Lambda}$ is a homeomorphism on $M_{(v)}^{1}\left(\mathbf{R}^{d}\right)$ when $\Lambda=\theta \mathbf{Z}^{d}$ and $\theta \in\left(0, \theta_{0}\right]$. The Gabor systems

$$
\left\{e^{i\langle\cdot, \iota\rangle} \phi(\cdot-j)\right\}_{(j, \iota) \in \Lambda} \quad \text { and } \quad\left\{e^{i\langle\cdot, \iota\rangle} \psi(\cdot-j)\right\}_{(j, \iota) \in \Lambda},
$$

are dual frames for $L^{2}\left(\mathbf{R}^{d}\right)$ when $\psi=\left(S_{\phi, \phi}^{\Lambda}\right)^{-1} \phi \in M_{(v)}^{1}\left(\mathbf{R}^{d}\right)$ and $\theta \in\left(0, \theta_{0}\right]$.

Let $v, \phi$ and $\Lambda$ be as in Proposition 2.5. Then $\left(S_{\phi, \phi}^{\Lambda}\right)^{-1} \phi$ is called the canonical dual window of $\phi$, with respect to $\Lambda$. We have

$$
S_{\phi, \phi}^{\Lambda}\left(e^{i\langle\cdot, \iota\rangle} f(\cdot-j)\right)=e^{i\langle\cdot, \iota\rangle}\left(S_{\phi, \phi}^{\Lambda} f\right)(\cdot-j),
$$

when $f \in M_{(1 / v)}^{\infty}\left(\mathbf{R}^{d}\right)$ and $(j, \iota) \in \Lambda$.

The next result concerns Gabor expansion of modulation spaces. It is a special case of [34 Theorem 3.7] (see also [17, Corollaries 12.2.5 and 12.2.6] and [11, Theorem 3.7]).

Proposition 2.6. Let $\theta>0, \Lambda=\theta \mathbf{Z}^{d}$,

$$
\Lambda^{2}=\Lambda \times \Lambda=\{(j, \iota)\}_{j, \iota \in \Lambda} \subseteq \mathbf{R}^{2 d},
$$

let $p, q, r \in(0, \infty]$ satisfy $r \leq \min (1, p, q)$, and let $\omega, v \in \mathscr{P}_{E}\left(\mathbf{R}^{2 d}\right)$ be such that $\omega$ is v-moderate. Suppose $\phi, \psi \in M_{(v)}^{r}\left(\mathbf{R}^{d}\right)$ are such that (2.11) are dual frames for 
$L^{2}\left(\mathbf{R}^{d}\right)$. Then the following is true:

(1) The operators

$$
C_{\phi}^{\Lambda}: M_{(\omega)}^{p, q}\left(\mathbf{R}^{d}\right) \mapsto \ell_{(\omega)}^{p, q}\left(\Lambda^{2}\right) \quad \text { and } \quad D_{\psi}^{\Lambda}: \ell_{(\omega)}^{p, q}\left(\Lambda^{2}\right) \mapsto M_{(\omega)}^{p, q}\left(\mathbf{R}^{d}\right)
$$

are continuous.

(2) The operators $S_{\phi, \psi} \equiv D_{\psi} \circ C_{\phi}$ and $S_{\psi, \phi} \equiv D_{\phi} \circ C_{\psi}$ are both the identity map on $M_{(\omega)}^{p, q}\left(\mathbf{R}^{d}\right)$, and if $f \in M_{(\omega)}^{p, q}\left(\mathbf{R}^{d}\right)$, then

$$
\begin{aligned}
f & =\sum_{j, \iota \in \Lambda} V_{\phi} f(j, \iota) e^{i\langle\cdot, \iota\rangle} \psi(\cdot-j) \\
& =\sum_{j, \iota \in \Lambda} V_{\psi} f(j, \iota) e^{i\langle\cdot, \iota\rangle} \phi(\cdot-j),
\end{aligned}
$$

with unconditional quasi-norm convergence in $M_{(\omega)}^{p, q}$ when $p, q<\infty$, and with convergence in $M_{(\omega)}^{\infty}$ with respect to the weak* topology otherwise.

(3) If $f \in M_{(1 / v)}^{\infty}\left(\mathbf{R}^{d}\right)$, then

$$
\|f\|_{M_{(\omega)}^{p, q}} \asymp\left\|V_{\phi} f\right\|_{\ell_{(\omega)}^{p, q}\left(\Lambda^{2}\right)} \asymp\left\|V_{\psi} f\right\|_{\ell_{(\omega)}^{p, q}\left(\Lambda^{2}\right)} .
$$

The series (2.12) are called Gabor expansions of $f$ with respect to $\phi, \psi$ and $\Lambda$.

Remark 2.7. There are many ways to achieve dual frames 2.11 satisfying the required properties in Proposition 2.6. In fact, let $v, v_{0} \in \mathscr{P}_{E}\left(\mathbf{R}^{2 d}\right)$ be submultiplicative such that $\omega$ is $v$-moderate and

$$
L_{\left(v_{0}\right)}^{1}\left(\mathbf{R}^{2 d}\right) \subseteq \bigcap_{0<r \leq 1} L^{r}\left(\mathbf{R}^{2 d}\right) .
$$

This inclusion is satisfied e.g. for $v_{0}(x)=e^{\varepsilon|x|}$ with $\varepsilon>0$. Proposition 2.5] guarantees that for some choice of $\phi, \psi \in M_{\left(v_{0} v\right)}^{1}\left(\mathbf{R}^{d}\right) \subseteq \bigcap_{0<r \leq 1} M_{(v)}^{r}\left(\mathbf{R}^{d}\right)$ and lattice $\Lambda \subseteq \mathbf{R}^{d}$, the sets in 2.11) where $\psi=\left(S_{\phi, \phi}^{\Lambda}\right)^{-1} \phi$, are dual frames.

We usually assume that $\Lambda=\theta \mathbf{Z}^{d}$, with $\theta>0$ small enough to guarantee the hypotheses in Propositions 2.5 and 2.6 be fulfilled, and that the window function and its dual belong to $M_{(v)}^{r}$ for every $r>0$. This is always possible, in view of Remark 2.7.

We need the following version of Proposition 2.5, which is a consequence of [3. Corollary 3.2] and the Fourier invariance of $\Sigma_{1}\left(\mathbf{R}^{2 d}\right)$.

Lemma 2.8. Suppose $v \in \mathscr{P}_{E}\left(\mathbf{R}^{4 d}\right)$ is submultiplicative, let $\phi_{1}, \phi_{2} \in \Sigma_{1}\left(\mathbf{R}^{d}\right) \backslash 0$, and let

$$
\Phi(x, \xi)=\phi_{1}(x) \overline{\widehat{\phi}_{2}(\xi)} e^{-i\langle x, \xi\rangle} .
$$


Then there is a lattice $\Lambda^{2} \subseteq \mathbf{R}^{2 d}$ such that

$$
\left\{\Phi(x-j, \xi-\iota) e^{i(\langle x, \kappa\rangle+\langle k, \xi\rangle)}\right\}_{(j, \iota),(k, \kappa) \in \Lambda^{2}}
$$

is a Gabor frame for $L^{2}\left(\mathbf{R}^{2 d}\right)$ with canonical dual frame

$$
\left\{\Psi(x-j, \xi-\iota) e^{i(\langle x, \kappa\rangle+\langle k, \xi\rangle)}\right\}_{(j, \iota),(k, \kappa) \in \Lambda^{2}},
$$

and

$$
\Psi=\left(S_{\Phi, \Phi}^{\Lambda^{2}}\right)^{-1} \Phi \in \bigcap_{r>0} M_{(v)}^{r}\left(\mathbf{R}^{2 d}\right) .
$$

The right-hand side of (2.13) is called the cross-Rihaczek distribution of $\phi_{1}$ and $\phi_{2}$ [17].

Remark 2.9. The last conclusion in Lemma 2.8 is a consequence of the sharper result [25, Lemma 2].

\subsection{Pseudodifferential operators and Gabor analysis}

In order to discuss a reformulation of pseudodifferential operators by means of Gabor analysis, we need the following matrix concepts [36].

Definition 2.10. Let $p, q \in(0, \infty], \theta>0$, let $J$ be an index set and let $\Lambda=\theta \mathbf{Z}^{d}$ be a lattice, and let $\omega \in \mathscr{P}_{E}\left(\mathbf{R}^{2 d}\right)$.

(1) $\mathbb{U}_{0}^{\prime}(J)$ is the set of all matrices $A=(\boldsymbol{a}(j, k))_{j, k \in J}$ with entries in $\mathbf{C}$;

(2) $\mathbb{U}_{0}(J)$ is the set of all $A=(\boldsymbol{a}(j, k))_{j, k \in J} \in \mathbb{U}_{0}^{\prime}(J)$ such that $\boldsymbol{a}(j, k) \neq 0$ for at most finitely many $(j, k) \in J \times J$

(3) for $A=(\boldsymbol{a}(j, k))_{j, k \in \Lambda} \in \mathbb{U}_{0}^{\prime}(\Lambda)$ let

$$
H_{A, \omega}(j, k)=\boldsymbol{a}(j, j-k) \omega(j, j-k) \quad \text { and } \quad h_{A, p, \omega}(k)=\left\|H_{A, \omega}(\cdot, k)\right\|_{\ell^{p}} .
$$

The set $\mathbb{U}^{p, q}(\omega, \Lambda)$ consists of all matrices $A=(\boldsymbol{a}(j, k))_{j, k \in \Lambda}$ such that

$$
\left\|(\boldsymbol{a}(j, k))_{j, k \in \Lambda}\right\|_{\mathbb{U}^{p, q}(\omega, \Lambda)} \equiv\left\|h_{A, p, \omega}\right\|_{\ell^{q}}
$$

is finite.

$\mathbb{U}^{p, q}(\omega, \Lambda)$ is a quasi-Banach space, and if $p, q \geq 1$ it is a Banach space.

If $J$ is an index set then $A=(\boldsymbol{a}(j, k))_{j, k \in J} \in \mathbb{U}_{0}^{\prime}(J)$ is called properly supported if the sets

$$
\left\{j \in J ; \boldsymbol{a}\left(j, k_{0}\right) \neq 0\right\} \quad \text { and } \quad\left\{k \in J ; \boldsymbol{a}\left(j_{0}, k\right) \neq 0\right\},
$$

are finite for every $j_{0}, k_{0} \in J$. The set of properly supported matrices is denoted $\mathbb{U}_{\mathrm{p}}(J)$, and evidently $\mathbb{U}_{0}(J) \subseteq \mathbb{U}_{\mathrm{p}}(J)$. The sets $\mathbb{U}_{0}(J)$ and $\mathbb{U}_{\mathrm{p}}(J)$ are rings under matrix multiplication, and $\mathbb{U}_{0}^{\prime}(J)$ is a $\mathbb{U}_{\mathrm{p}}(J)$-module with respect to matrix multiplication. 
Let $\phi_{1}, \phi_{2} \in \Sigma_{1}\left(\mathbf{R}^{d}\right) \backslash 0$, let $\Phi$ be defined by (2.13), let $\Lambda \subseteq \mathbf{R}^{d}$ be a lattice such that $\Lambda^{2}=\Lambda \times \Lambda \subseteq \mathbf{R}^{2 d}$ makes (2.14) a Gabor frame in accordance with Lemma 2.8 and let $\Psi=\left(S_{\Phi \Phi}^{\Lambda^{2}}\right)^{-1} \Phi$ be the canonical dual window of $\Phi$. Suppose $\omega_{0} \in \mathscr{P}_{E}\left(\mathbf{R}^{4 d}\right)$ and set

$$
\omega(x, \xi y, \eta)=\omega_{0}(x, \eta, \xi-\eta, y-x) .
$$

Let $a \in M_{\left(\omega_{0}\right)}^{p, q}\left(\mathbf{R}^{2 d}\right)$, define

$$
\boldsymbol{a}(\boldsymbol{j}, \boldsymbol{k})=V_{\Psi} a(j, \kappa, \iota-\kappa, k-j) e^{i\langle k-j, \kappa\rangle},
$$

where

$$
\boldsymbol{j}=(j, \iota) \in \Lambda^{2} \quad \text { and } \quad \boldsymbol{k}=(k, \kappa) \in \Lambda^{2},
$$

and define the matrix

$$
A=(\boldsymbol{a}(\boldsymbol{j}, \boldsymbol{k}))_{\boldsymbol{j}, \boldsymbol{k} \in \Lambda^{2}} .
$$

Then it follows from Propositions 2.5 and 2.6 that

$$
\|a\|_{M_{\left(\omega_{0}\right)}^{p, q}} \asymp\|A\|_{\mathbb{U}^{p, q}\left(\omega, \Lambda^{2}\right)},
$$

provided $\theta$ is sufficiently small.

By identifying matrices with corresponding linear operators, [35, Lemma 3.3] gives

$$
\operatorname{Op}(a)=D_{\phi_{1}} \circ A \circ C_{\phi_{2}}
$$

Hence, if $b \in \mathcal{S}_{1 / 2}\left(\mathbf{R}^{2 d}\right)$,

$$
\begin{aligned}
\boldsymbol{b}(\boldsymbol{j}, \boldsymbol{k}) & =V_{\Psi} b(j, \kappa, \iota-\kappa, k-j) e^{i\langle k-j, \kappa\rangle}, \quad \boldsymbol{j}, \boldsymbol{k} \in \Lambda^{2}, \\
B & =(\boldsymbol{b}(\boldsymbol{j}, \boldsymbol{k}))_{\boldsymbol{j}, \boldsymbol{k} \in \Lambda^{2}},
\end{aligned}
$$

and the matrix $C$ is defined as

$$
C=C_{\phi_{2}} \circ D_{\phi_{1}}
$$

then

$$
\mathrm{Op}\left(a \#_{0} b\right)=\mathrm{Op}(a) \circ \mathrm{Op}(b)=D_{\phi_{1}} \circ(A \circ C \circ B) \circ C_{\phi_{2}},
$$

and

$$
\left\|a \#_{0} b\right\|_{M_{\left(\omega_{0}\right)}^{p, q}} \asymp\|A \circ C \circ B\|_{\mathbb{U}^{p, q}\left(\omega, \Lambda^{2}\right)} .
$$

\subsection{Composition of pseudodifferential operators with symbols in Banach modulation spaces}

We recall algebraic results for pseudodifferential operators with symbols in modulation spaces with Lebesgue exponents not smaller than one [7] 23,36 . 
If $A \in \mathbf{M}(d, \mathbf{R})$, then the product $\#_{A}$ with $N$ factors

$$
\left(a_{1}, \ldots, a_{N}\right) \mapsto a_{1} \#_{A} \cdots \#_{A} a_{N}
$$

from $\mathcal{S}_{1 / 2}\left(\mathbf{R}^{2 d}\right) \times \cdots \times \mathcal{S}_{1 / 2}\left(\mathbf{R}^{2 d}\right)$ to $\mathcal{S}_{1 / 2}\left(\mathbf{R}^{2 d}\right)$ is defined by the formula

$$
\mathrm{Op}_{A}\left(a_{1} \#_{A} \cdots \#_{A} a_{N}\right)=\mathrm{Op}_{A}\left(a_{1}\right) \circ \cdots \circ \mathrm{Op}_{A}\left(a_{N}\right) .
$$

The map (2.24) can be extended in different ways, e.g. as in [7, Theorem 2.11] which is stated in a generalized form in Theorem 2.11 below. Assume that the weight functions satisfy

$$
\omega_{0}\left(T_{A}\left(X_{N}, X_{0}\right)\right) \lesssim \prod_{j=1}^{N} \omega_{j}\left(T_{A}\left(X_{j}, X_{j-1}\right)\right), \quad X_{0}, \ldots, X_{N} \in \mathbf{R}^{2 d},
$$

where

$$
\begin{aligned}
T_{A}(X, Y) & =\left(y+A(x-y), \xi+A^{*}(\eta-\xi), \eta-\xi, x-y\right), \\
X & =(x, \xi) \in \mathbf{R}^{2 d}, \quad Y=(y, \eta) \in \mathbf{R}^{2 d} .
\end{aligned}
$$

Here $A^{*}$ denotes $A$ transposed. Assume that the Lebesgue parameters satisfy

$$
\max \left(\mathrm{R}_{N}\left(\boldsymbol{q}^{\prime}\right), 0\right) \leq \min _{j=1, \ldots, N}\left(\frac{1}{p_{0}^{\prime}}, \frac{1}{q_{0}}, \frac{1}{p_{j}}, \frac{1}{q_{j}^{\prime}}, \mathrm{R}_{N}(\boldsymbol{p})\right)
$$

or

$$
\mathrm{R}_{N}(\boldsymbol{p}) \geq 0, \quad \frac{1}{q_{0}} \leq \frac{1}{p_{0}^{\prime}} \leq \frac{1}{2} \quad \text { and } \quad \frac{1}{q_{j}^{\prime}} \leq \frac{1}{p_{j}} \leq \frac{1}{2}, \quad j=1, \ldots, N
$$

where

$$
\begin{aligned}
\mathrm{R}_{N}(\boldsymbol{p}) & =(N-1)^{-1}\left(\sum_{j=1}^{N} \frac{1}{p_{j}}-\frac{1}{p_{0}}\right), \\
\boldsymbol{p} & =\left(p_{0}, p_{1}, \ldots, p_{N}\right) \in[1, \infty]^{N+1} .
\end{aligned}
$$

Theorem 2.11. Suppose $p_{j}, q_{j} \in[1, \infty], j=0,1, \ldots, N$ satisfy (2.27) or (2.28), and suppose $\omega_{j} \in \mathscr{P}_{E}\left(\mathbf{R}^{4 d}\right), j=0,1, \ldots, N$, satisfy (2.25) and (2.26). Then the map (2.24) from $\mathcal{S}_{1 / 2}\left(\mathbf{R}^{2 d}\right) \times \cdots \times \mathcal{S}_{1 / 2}\left(\mathbf{R}^{2 d}\right)$ to $\mathcal{S}_{1 / 2}\left(\mathbf{R}^{2 d}\right)$ extends uniquely to a continuous and associative map from $M_{\left(\omega_{1}\right)}^{p_{1}, q_{1}}\left(\mathbf{R}^{2 d}\right) \times \cdots \times M_{\left(\omega_{N}\right)}^{p_{N}, q_{N}}\left(\mathbf{R}^{2 d}\right)$ to $M_{\left(\omega_{0}\right)}^{p_{0}, q_{0}}\left(\mathbf{R}^{2 d}\right)$, and

$$
\left\|a_{1} \#_{A} \cdots \#_{A} a_{N}\right\|_{M_{\left(\omega_{0}\right)}^{p_{0}, q_{0}}} \lesssim \prod_{j=1}^{N}\left\|a_{j}\right\|_{M_{\left(\omega_{j}\right)}^{p_{j}, q_{j}}}
$$

for $a_{j} \in M_{\left(\omega_{j}\right)}^{p_{j}, q_{j}}\left(\mathbf{R}^{2 d}\right), j=1, \ldots, N$. 
Theorem 2.11 follows by similar arguments as in the proof of [7, Theorem 2.11]. The details are left for the reader.

Remark 2.12. We note that the definition of $T_{A}$ in 7 , Eq. (2.30)] is incorrect and should be replaced by (2.26) with $A=t I$, in order for [7, Theorem 2.11] to hold. A corrected version of [7] has been posted on arXiv.

\section{Composition of Pseudodifferential Operators with Symbols in Quasi-Banach Modulation Spaces}

In this section, we deduce a composition result for pseudodifferential operators with symbols in modulation spaces with Lebesgue parameters in $(0, \infty]$.

If $A \in \mathbf{M}(d, \mathbf{R})$, then the map

$$
\left(a_{1}, a_{2}\right) \mapsto a_{1} \#_{A} a_{2}
$$

from $\mathcal{S}_{1 / 2}\left(\mathbf{R}^{2 d}\right) \times \mathcal{S}_{1 / 2}\left(\mathbf{R}^{2 d}\right)$ to $\mathcal{S}_{1 / 2}\left(\mathbf{R}^{2 d}\right)$ is defined by

$$
\mathrm{Op}_{A}\left(a_{1} \#_{A} a_{2}\right)=\mathrm{Op}_{A}\left(a_{1}\right) \circ \mathrm{Op}_{A}\left(a_{2}\right) .
$$

The following result is the principal result of this paper. It concerns sufficient conditions for the unique extension of (3.1) to symbols in quasi-Banach modulation spaces.

The weight functions are assumed to obey the estimates

$$
\omega_{0}\left(T_{A}(Z, X)\right) \lesssim \omega_{1}\left(T_{A}(Y, X)\right) \omega_{2}\left(T_{A}(Z, Y)\right), \quad X, Y, Z \in \mathbf{R}^{2 d},
$$

where

$$
\begin{aligned}
T_{A}(X, Y) & =\left(y+A(x-y), \xi+A^{*}(\eta-\xi), \eta-\xi, x-y\right), \\
X & =(x, \xi) \in \mathbf{R}^{2 d}, \quad Y=(y, \eta) \in \mathbf{R}^{2 d}
\end{aligned}
$$

(cf. 2.25) and (2.26) ).

Theorem 3.1. Let $A \in \mathbf{M}(d, \mathbf{R})$ and suppose $\omega_{j} \in \mathscr{P}_{E}\left(\mathbf{R}^{4 d}\right), j=0,1,2$, satisfy (3.2) and (3.3). Suppose $p_{j}, q_{j} \in(0, \infty], j=0,1,2$, satisfy

$$
\frac{1}{p_{0}} \leq \frac{1}{p_{1}}+\frac{1}{p_{2}}
$$

and either

$$
q_{1}, q_{2} \leq q_{0} \leq \min \left(1, p_{0}\right)
$$

or

$$
\min \left(1, p_{0}\right) \leq q_{1}, q_{2} \leq q_{0} \quad \text { and } \quad \frac{1}{\min \left(1, p_{0}\right)}+\frac{1}{q_{0}} \leq \frac{1}{q_{1}}+\frac{1}{q_{2}}
$$


Then the map $\left(a_{1}, a_{2}\right) \mapsto a_{1} \#_{A} a_{2}$ from $\mathcal{S}_{1 / 2}\left(\mathbf{R}^{2 d}\right) \times \mathcal{S}_{1 / 2}\left(\mathbf{R}^{2 d}\right)$ to $\mathcal{S}_{1 / 2}\left(\mathbf{R}^{2 d}\right)$ extends uniquely to a continuous map from $M_{\left(\omega_{1}\right)}^{p_{1}, q_{1}}\left(\mathbf{R}^{2 d}\right) \times M_{\left(\omega_{2}\right)}^{p_{2}, q_{2}}\left(\mathbf{R}^{2 d}\right)$ to $M_{\left(\omega_{0}\right)}^{p_{0}, q_{0}}\left(\mathbf{R}^{2 d}\right)$, and

$$
\left\|a_{1} \#_{A} a_{2}\right\|_{M_{\left(\omega_{0}\right)}^{p_{0}, q_{0}}} \lesssim\left\|a_{1}\right\|_{M_{\left(\omega_{1}\right)}^{p_{1}, q_{1}}}\left\|a_{2}\right\|_{M_{\left(\omega_{2}\right)}^{p_{2}, q_{2}}}
$$

for all $a_{1} \in M_{\left(\omega_{1}\right)}^{p_{1}, q_{1}}\left(\mathbf{R}^{2 d}\right)$ and $a_{2} \in M_{\left(\omega_{2}\right)}^{p_{2}, q_{2}}\left(\mathbf{R}^{2 d}\right)$.

We need some preparations for the proof. The following result contains the needed continuity properties for matrix operators.

Proposition 3.2. Let $\Lambda \subseteq \mathbf{R}^{d}$ be a lattice, let $p_{j}, q_{j} \in(0, \infty], j=0,1,2$, be such that (3.4)-(3.6) hold, and suppose $\omega_{0}, \omega_{1}, \omega_{2} \in \mathscr{P}_{E}\left(\mathbf{R}^{2 d}\right)$ satisfy

$$
\omega_{0}(x, z) \lesssim \omega_{1}(x, y) \omega_{2}(y, z), \quad x, y, z \in \mathbf{R}^{d} .
$$

Then the map $\left(A_{1}, A_{2}\right) \mapsto A_{1} \circ A_{2}$ from $\mathbb{U}_{0}(\Lambda) \times \mathbb{U}_{0}(\Lambda)$ to $\mathbb{U}_{0}(\Lambda)$ extends uniquely to a continuous map from $\mathbb{U}^{p_{1}, q_{1}}\left(\omega_{1}, \Lambda\right) \times \mathbb{U}^{p_{2}, q_{2}}\left(\omega_{2}, \Lambda\right)$ to $\mathbb{U}^{p_{0}, q_{0}}\left(\omega_{0}, \Lambda\right)$, and

$$
\left\|A_{1} \circ A_{2}\right\|_{\mathbb{U}^{p_{0}, q_{0}}\left(\omega_{0}, \Lambda\right)} \lesssim\left\|A_{1}\right\|_{\mathbb{U}^{p_{1}, q_{1}}\left(\omega_{1}, \Lambda\right)}\left\|A_{2}\right\|_{\mathbb{U}^{p_{2}, q_{2}}\left(\omega_{2}, \Lambda\right)} .
$$

Proof. Let $\mathbb{U}_{0,+}^{\prime}(\Lambda)$ be the set of all $A \in \mathbb{U}_{0}^{\prime}(\Lambda)$ with non-negative entries, let $A_{m}=\left(\boldsymbol{a}_{m}(j, k)\right)_{j, k \in \Lambda} \in \mathbb{U}_{0}(\Lambda) \cap \mathbb{U}_{0,+}^{\prime}(\Lambda), m=1,2$, denote the matrix elements of $B=A_{1} \circ A_{2}$ by $\boldsymbol{b}(j, k), j, k \in \Lambda$, and set $p=p_{0}, q=q_{0}, \omega=\omega_{0}$,

$$
\mathfrak{a}_{m}(j, k) \equiv\left|\boldsymbol{a}_{m}(j, j-k)\right| \omega_{m}(j, j-k) \quad \text { and } \quad \mathfrak{b}(j, k) \equiv|\boldsymbol{b}(j, j-k)| \omega(j, j-k),
$$

$m=1,2$. Then

$$
\begin{aligned}
\left\|A_{m}\right\|_{\mathbb{U}^{p^{m}}, q_{m}}\left(\omega_{m}, \Lambda\right) & =\left\|\mathfrak{a}_{m}\right\|_{\ell^{p_{m}, q_{m}}}, \quad m=1,2, \\
\left\|A_{1} \circ A_{2}\right\|_{\mathbb{U}^{p, q}(\omega, \Lambda)} & =\|\mathfrak{b}\|_{\ell^{p, q}},
\end{aligned}
$$

and we first prove

$$
\|\mathfrak{b}\|_{\ell^{p, q}} \leq\left\|\mathfrak{a}_{1}\right\|_{\ell^{p_{1}, q_{1}}}\left\|\mathfrak{a}_{2}\right\|_{\ell^{p_{2}, q_{2}}}
$$

We have

$$
\mathfrak{b}(j, k) \leq \sum_{l \in \Lambda} \mathfrak{a}_{1}(j, l) \mathfrak{a}_{2}(j-l, k-l) .
$$

In order to estimate $\|\mathfrak{b}(\cdot, k)\|_{\ell^{p}}$ we consider the cases $p<1$ and $p \geq 1$ separately.

First assume that $p<1$, and set $r_{j}=\frac{p_{j}}{p}$. Then $\frac{1}{r_{1}}+\frac{1}{r_{2}} \geq 1$ by assumption (3.4), and therefore Hölder's inequality yields for $k \in \Lambda$

$$
\begin{aligned}
\|\mathfrak{b}(\cdot, k)\|_{\ell^{p}}^{p} & \leq \sum_{j \in \Lambda}\left(\sum_{l \in \Lambda} \mathfrak{a}_{1}(j, l) \mathfrak{a}_{2}(j-l, k-l)\right)^{p} \\
& \leq \sum_{l \in \Lambda} \sum_{j \in \Lambda}\left(\mathfrak{a}_{1}(j, l) \mathfrak{a}_{2}(j-l, k-l)\right)^{p}
\end{aligned}
$$




$$
\begin{aligned}
& \leq \sum_{l \in \Lambda}\left\|\mathfrak{a}_{1}(\cdot, l)^{p}\right\|_{\ell^{r_{1}}}\left\|\mathfrak{a}_{2}(\cdot, k-l)^{p}\right\|_{\ell^{r_{2}}} \\
& =\sum_{l \in \Lambda}\left\|\mathfrak{a}_{1}(\cdot, l)\right\|_{\ell^{p_{1}}}^{p}\left\|\mathfrak{a}_{2}(\cdot, k-l)\right\|_{\ell^{p_{2}}}^{p},
\end{aligned}
$$

that is

$$
\|\mathfrak{b}(\cdot, k)\|_{\ell^{p}} \leq\left(c_{1} * c_{2}(k)\right)^{\frac{1}{p}},
$$

with $c_{m}(k)=\left\|\mathfrak{a}_{m}(\cdot, k)\right\|_{\ell^{p_{m}}}^{p}, m=1,2$.

In order to estimate $\left(c_{1} * c_{2}\right)^{\frac{1}{p}}$ we first assume (3.5). Then

$$
\begin{aligned}
\|\mathfrak{b}\|_{\ell^{p, q}} & \leq\left\|\left(c_{1} * c_{2}\right)^{\frac{1}{p}}\right\|_{\ell^{q}}=\left\|c_{1} * c_{2}\right\|_{\ell^{q} / p}^{\frac{1}{p}} \\
& \leq\left(\left\|c_{1}\right\|_{\ell^{q / p}}\left\|c_{2}\right\|_{\ell q / p}\right)^{\frac{1}{p}}=\left\|\mathfrak{a}_{1}\right\|_{\ell^{p_{1}, q}}\left\|\mathfrak{a}_{2}\right\|_{\ell^{p_{2}, q}} \leq\left\|\mathfrak{a}_{1}\right\|_{\ell^{p_{1}, q_{1}}}\left\|\mathfrak{a}_{2}\right\|_{\ell^{p_{2}, q_{2}}},
\end{aligned}
$$

and the result follows in this case.

If instead (3.6) holds then $q \geq q_{1}, q_{2} \geq p$, and $r_{j}=q_{j} / p, j=1,2$, and $r=q / p$ satisfy

$$
r_{1}, r_{2}, r \geq 1 \text { and } \frac{1}{r_{1}}+\frac{1}{r_{2}} \geq 1+\frac{1}{r} .
$$

Hence Young's inequality may be applied and gives

$$
\|\mathfrak{b}\|_{\ell^{p, q}} \leq\left\|c_{1} * c_{2}\right\|_{\ell^{r}}^{\frac{1}{p}} \leq\left(\left\|c_{1}\right\|_{\ell^{r_{1}}}\left\|c_{2}\right\|_{\ell^{r_{2}}}\right)^{\frac{1}{p}}=\left\|\mathfrak{a}_{1}\right\|_{\ell^{p_{1}, q_{1}}}\left\|\mathfrak{a}_{2}\right\|_{\ell^{p_{2}, q_{2}}},
$$

and the result follows in this case as well.

Next we consider the case $p \geq 1$. By Minkowski's and Hölder's inequalities and the assumption (3.4) we get from (3.8)

$$
\begin{aligned}
\|\mathfrak{b}(\cdot, k)\|_{\ell^{p}} & \leq \sum_{l \in \Lambda}\left\|\mathfrak{a}_{1}(\cdot, l) \mathfrak{a}_{2}(\cdot-l, k-l)\right\|_{\ell^{p}} \\
& \leq \sum_{l \in \Lambda}\left\|\mathfrak{a}_{1}(\cdot, l)\right\|_{\ell^{p_{1}}}\left\|\mathfrak{a}_{2}(\cdot, k-l)\right\|_{\ell^{p_{2}}}=c_{1} * c_{2}(k),
\end{aligned}
$$

where $c_{m}(k)=\left\|\mathfrak{a}_{m}(\cdot, k)\right\|_{\ell^{p_{m}}}, m=1,2$.

If (3.6) holds then $q \geq q_{1}, q_{2} \geq 1$ and Young's inequality gives

$$
\|\mathfrak{b}\|_{\ell^{p, q}} \leq\left\|c_{1} * c_{2}\right\|_{\ell^{q}} \leq\left\|c_{1}\right\|_{\ell^{q_{1}}}\left\|c_{2}\right\|_{\ell^{q_{2}}}=\left\|\mathfrak{a}_{1}\right\|_{\ell^{p_{1}, q_{1}}}\left\|\mathfrak{a}_{2}\right\|_{\ell^{p_{2}, q_{2}}}
$$

and the result follows. If instead (3.5) holds then $q \leq 1$ and (3.9) gives

$$
\begin{aligned}
\|\mathfrak{b}\|_{\ell^{p, q}} & \leq\left\|c_{1} * c_{2}\right\|_{\ell^{q}} \leq\left\|c_{1}\right\|_{\ell^{q}}\left\|c_{2}\right\|_{\ell^{q}}=\left\|\mathfrak{a}_{1}\right\|_{\ell^{p_{1}, q}}\left\|\mathfrak{a}_{2}\right\|_{\ell^{p_{2}, q}} \\
& \leq\left\|\mathfrak{a}_{1}\right\|_{\ell^{p_{1}, q_{1}}}\left\|\mathfrak{a}_{2}\right\|_{\ell^{p_{2}, q_{2}}} .
\end{aligned}
$$

Thus we have proved (3.7) when $A_{1}, A_{2} \in \mathbb{U}_{0}(\Lambda) \cap \mathbb{U}_{0,+}^{\prime}(\Lambda)$.

By Beppo-Levi's theorem or Fatou's lemma applied to the previous situation, we obtain that $A_{1} \circ A_{2}$ is uniquely defined as an element in $\mathbb{U}^{p, q}(\omega, \Lambda)$ and (3.7) holds, provided $A_{m} \in \mathbb{U}^{p_{m}, q_{m}}\left(\omega_{m}, \Lambda\right) \cap \mathbb{U}_{0,+}^{\prime}(\Lambda)$ for $m=1,2$. 
For $A_{m} \in \mathbb{U}^{p_{m}, q_{m}}\left(\omega_{m}, \Lambda\right), m=1,2$, there are unique

$$
A_{m, k} \in \mathbb{U}^{p_{m}, q_{m}}\left(\omega_{m}, \Lambda\right) \cap \mathbb{U}_{0,+}^{\prime}(\Lambda), \quad m=1,2, \quad k=1, \ldots, 4,
$$

such that

$$
A_{m}=\sum_{k=1}^{4} i^{k} A_{m, k}
$$

and we have

$$
\left\|A_{m, k}\right\|_{\mathbb{U}^{p_{m}}, q_{m}}\left(\omega_{m}, \Lambda\right) \leq\left\|A_{m}\right\|_{\mathbb{U}^{p_{m}}, q_{m}\left(\omega_{m}, \Lambda\right)}, \quad m=1,2, \quad k=1, \ldots, 4 .
$$

Since the assertion holds true for $A_{1, k}$ and $A_{2, l}$ in place of $A_{1}$ and $A_{2}$, it follows from the latter estimate that

$$
A_{1} \circ A_{2}=\sum_{k, l=1}^{4} i^{k+l} A_{1, k} \circ A_{2, l} \in \mathbb{U}^{p, q}(\omega, \Lambda),
$$

is uniquely defined and that (3.7) holds for $A_{m} \in \mathbb{U}^{p_{m}}, q_{m}\left(\omega_{m}, \Lambda\right), m=1,2$.

We also need the following result on the composition of the analysis operator and the synthesis operator defined by two Gabor systems.

Lemma 3.3. Suppose $\Lambda \subseteq \mathbf{R}^{d}$ is a lattice, $\Lambda^{2}=\Lambda \times \Lambda$ and $\phi_{1}, \phi_{2} \in \Sigma_{1}\left(\mathbf{R}^{d}\right) \backslash 0$. Let $C_{\phi_{2}}=C_{\phi_{2}}^{\Lambda}$ be the Gabor analysis operator and let $D_{\phi_{1}}=D_{\phi_{1}}^{\Lambda}$ be the Gabor synthesis operator defined by $\phi_{2}$ and $\phi_{1}$, respectively, and $\Lambda$. Then $C_{\phi_{2}} \circ D_{\phi_{1}}$ is the matrix $(\boldsymbol{c}(\boldsymbol{j}, \boldsymbol{k}))_{\boldsymbol{j}, \boldsymbol{k} \in \Lambda^{2}}$ where

$$
\boldsymbol{c}(\boldsymbol{j}, \boldsymbol{k})=e^{i\langle k, \kappa-\iota\rangle} V_{\phi_{2}} \phi_{1}(\boldsymbol{j}-\boldsymbol{k}), \quad \boldsymbol{j}=(j, \iota), \quad \boldsymbol{k}=(k, \kappa) .
$$

If $\omega_{0}(X, Y)=\omega(X-Y), X, Y \in \mathbf{R}^{2 d}$ for $\omega \in \mathscr{P}_{E}\left(\mathbf{R}^{2 d}\right)$, then

$$
(\boldsymbol{c}(\boldsymbol{j}, \boldsymbol{k}))_{\boldsymbol{j}, \boldsymbol{k} \in \Lambda^{2}} \in \bigcap_{\substack{q>0 \\ \omega \in \mathscr{P}_{E}\left(\mathbf{R}^{2 d}\right)}} \mathbb{U}^{\infty, q}\left(\omega_{0}, \Lambda^{2}\right) .
$$

Proof. Let $f$ be a sequence on $\Lambda^{2}$ such that $f(\boldsymbol{k}) \neq 0$ for at most a finite number of $\boldsymbol{k} \in \Lambda^{2}$. Then

$$
D_{\phi_{1}} f=\sum_{\boldsymbol{k} \in \Lambda^{2}} f(\boldsymbol{k}) \phi_{1, \boldsymbol{k}}, \quad \phi_{1, \boldsymbol{k}} \equiv \phi_{1}(\cdot-k) e^{i\langle\cdot \kappa\rangle}, \quad \boldsymbol{k}=(k, \kappa),
$$

and

$$
C_{\phi_{2}}\left(D_{\phi_{1}} f\right)(\boldsymbol{j})=V_{\phi_{2}}\left(D_{\phi_{1}} f\right)(\boldsymbol{j})=\sum_{\boldsymbol{k} \in \Lambda^{2}} V_{\phi_{2}} \phi_{1, \boldsymbol{k}}(\boldsymbol{j}) f(\boldsymbol{k}) .
$$

If $\boldsymbol{j}=(j, \iota)$ then $C_{\phi_{2}} \circ D_{\phi_{1}}$ is hence given by the matrix $C=(\boldsymbol{c}(\boldsymbol{j}, \boldsymbol{k}))_{\boldsymbol{j}, \boldsymbol{k} \in \Lambda^{2}}$ where

$$
\boldsymbol{c}(\boldsymbol{j}, \boldsymbol{k})=V_{\phi_{2}} \phi_{1, \boldsymbol{k}}(\boldsymbol{j})=(2 \pi)^{-\frac{d}{2}} \int_{\mathbf{R}^{d}} \phi_{1, \boldsymbol{k}}(y) \overline{\phi_{2}(y-j)} e^{-i\langle y, \iota\rangle} d y
$$




$$
\begin{aligned}
& =(2 \pi)^{-\frac{d}{2}} e^{i\langle k, \kappa-\iota\rangle} \int_{\mathbf{R}^{d}} \phi_{1}(y) \overline{\phi_{2}(y-(j-k))} e^{-i\langle y, \iota-\kappa\rangle} d y \\
& =e^{i\langle k, \kappa-\iota\rangle} V_{\phi_{2}} \phi_{1}(\boldsymbol{j}-\boldsymbol{k})
\end{aligned}
$$

which proves (3.10).

It remains to prove (3.11). Let $\omega \in \mathscr{P}_{E}\left(\mathbf{R}^{2 d}\right)$ and $q>0$. Since $\phi_{1}, \phi_{2} \in \Sigma_{1}\left(\mathbf{R}^{d}\right)$, we have by [33, Theorem 2.4]

$$
\left|V_{\phi_{2}} \phi_{1}(x, \xi)\right| \lesssim e^{-r(|x|+|\xi|)},
$$

for every $r>0$. From (3.10) and (2.15) we obtain

$$
h_{C, \infty, \omega_{0}}(\boldsymbol{k})=\sup _{\boldsymbol{j} \in \Lambda^{2}}\left|H_{C, \omega_{0}}(\boldsymbol{j}, \boldsymbol{k})\right|=\left|V_{\phi_{2}} \phi_{1}(\boldsymbol{k}) \omega(\boldsymbol{k})\right| .
$$

A combination of these relations and (2.16) now give

$$
\|C\|_{\mathbb{U}^{\infty}, q\left(\omega_{0}, \Lambda^{2}\right)}=\left\|h_{C, \infty, \omega_{0}}\right\|_{\ell q}=\left\|V_{\phi_{2}} \phi_{1} \cdot \omega\right\|_{\ell q\left(\Lambda^{2}\right)}<\infty .
$$

Hence $C \in \mathbb{U}^{\infty, q}\left(\omega_{0}, \Lambda^{2}\right)$ for any $\omega \in \mathscr{P}_{E}\left(\mathbf{R}^{2 d}\right)$ and any $q>0$.

Proof of Theorem [3.1, By [36] Proposition 2.8] and Proposition [2.1] we may assume that $A=0$. Pick $\phi_{1}, \phi_{2} \in \Sigma_{1}\left(\mathbf{R}^{d}\right) \backslash 0$ and a lattice $\Lambda \subseteq \mathbf{R}^{d}$ such that $\Phi, \Psi$ and $\Lambda^{2}=\Lambda \times \Lambda \subseteq \mathbf{R}^{2 d}$ are as in Lemma 2.8. Let finally $a_{m} \in M_{\left(\omega_{m}\right)}^{p_{m}, q_{m}}\left(\mathbf{R}^{2 d}\right)$, $m=1,2$.

By 2.17)-2.20, we have for $m=1,2$

$$
\left\|a_{m}\right\|_{M_{\left(\omega_{m}\right)}^{p_{m}, q_{m}}} \asymp\left\|A_{m}\right\|_{\mathbb{U}^{p_{m}, q_{m}}\left(\vartheta_{m}, \Lambda^{2}\right)},
$$

and

$$
\operatorname{Op}\left(a_{m}\right)=D_{\phi_{1}} \circ A_{m} \circ C_{\phi_{2}},
$$

where

$$
\begin{aligned}
A_{m} & =\left(\boldsymbol{a}_{m}(\boldsymbol{j}, \boldsymbol{k})\right)_{\boldsymbol{j}, \boldsymbol{k} \in \Lambda^{2}}, \\
\boldsymbol{a}_{m}(\boldsymbol{j}, \boldsymbol{k}) & \equiv e^{i\langle k-j, \kappa\rangle} V_{\Psi} a_{m}(j, \kappa, \iota-\kappa, k-j), \quad \boldsymbol{j}=(j, \iota), \quad \boldsymbol{k}=(k, \kappa) \in \Lambda^{2},
\end{aligned}
$$

and

$$
\vartheta_{m}(x, \xi, y, \eta)=\omega_{m}(x, \eta, \xi-\eta, y-x) .
$$

Condition (3.2) means for the weights $\vartheta_{m}, m=0,1,2$,

$$
\vartheta_{0}(X, Y) \lesssim \vartheta_{1}(X, Z) \vartheta_{2}(Z, Y), \quad X, Y, Z \in \mathbf{R}^{2 d} .
$$

Pick $v_{1} \in \mathscr{P}_{E}\left(\mathbf{R}^{d}\right)$ even so that $\omega_{2}$ is $v_{2}$-moderate with

$$
v_{2}=v_{1} \otimes v_{1} \otimes v_{1} \otimes v_{1} \in \mathscr{P}_{E}\left(\mathbf{R}^{4 d}\right),
$$

set $v=v_{1}^{2} \otimes v_{1} \in \mathscr{P}_{E}\left(\mathbf{R}^{2 d}\right)$ and

$$
v_{0}(X, Y)=v(X-Y) \in \mathscr{P}_{E}\left(\mathbf{R}^{4 d}\right), \quad X, Y \in \mathbf{R}^{2 d} .
$$


Then $v_{0}$ is designed to guarantee

$$
\vartheta_{2}(X, Y) \lesssim v_{0}(X, Z) \vartheta_{2}(Z, Y), \quad X, Y, Z \in \mathbf{R}^{2 d} .
$$

We have by (2.21) and (2.22)

$$
\mathrm{Op}\left(a_{1}\right) \circ \mathrm{Op}\left(a_{2}\right)=D_{\phi_{1}} \circ A \circ C_{\phi_{2}},
$$

where

$$
A=A_{1} \circ C \circ A_{2}
$$

and $C=C_{\phi_{2}} \circ D_{\phi_{1}}$. By Lemma 3.3

$$
C \in \bigcap_{r>0} \mathbb{U}^{\infty, r}\left(v_{0}, \Lambda^{2}\right) .
$$

Set $r=\min \left(1, p_{2}, q_{2}\right)$. Then we obtain from 2.23, 3.14 , 3.15 and Proposition 3.2 applied twice

$$
\begin{aligned}
\left\|a_{1} \#_{0} a_{2}\right\|_{M_{\left(\omega_{0}\right)}^{p_{0}, q_{0}}} & \asymp\left\|A_{1} \circ C \circ A_{2}\right\|_{\mathbb{U}^{p_{0}, q_{0}}\left(\vartheta_{0}, \Lambda^{2}\right)} \\
& \lesssim\left\|A_{1}\right\|_{\mathbb{U}^{p_{1}, q_{1}}\left(\vartheta_{1}, \Lambda^{2}\right)}\left\|C \circ A_{2}\right\|_{\mathbb{U}^{p_{2}, q_{2}}\left(\vartheta_{2}, \Lambda^{2}\right)} \\
& \lesssim\left\|A_{1}\right\|_{\mathbb{U}^{p_{1}, q_{1}}\left(\vartheta_{1}, \Lambda^{2}\right)}\|C\|_{\mathbb{U}^{\infty, r}\left(v_{0}, \Lambda^{2}\right)}\left\|A_{2}\right\|_{\mathbb{U}^{p_{2}, q_{2}}\left(\vartheta_{2}, \Lambda^{2}\right)} \\
& \asymp\left\|A_{1}\right\|_{\mathbb{U}^{p_{1}, q_{1}}\left(\vartheta_{1}, \Lambda^{2}\right)}\left\|A_{2}\right\|_{\mathbb{U}^{p_{2}, q_{2}}\left(\vartheta_{2}, \Lambda^{2}\right)} \\
& \asymp\left\|a_{1}\right\|_{M_{\left(\omega_{1}\right)}^{p_{1}, q_{1}}}\left\|a_{2}\right\|_{M_{\left(\omega_{2}\right)}^{p_{2}, q_{2}}} .
\end{aligned}
$$

It remains to prove the claimed uniqueness of the extension. If (3.5) holds then $M_{\left(\omega_{j}\right)}^{p_{j}, q_{j}} \subseteq M_{\left(\omega_{j}\right)}^{\infty, 1}, j=1,2$, and $M_{\left(\omega_{0}\right)}^{p_{0}, q_{0}} \subseteq M_{\left(\omega_{0}\right)}^{\infty, 1}$. Then the claim follows from the uniqueness of the extension

$$
M_{\left(\omega_{1}\right)}^{\infty, 1} \#_{A} M_{\left(\omega_{2}\right)}^{\infty, 1} \subseteq M_{\left(\omega_{0}\right)}^{\infty, 1},
$$

which is proved in [7. Theorem 2.11].

Suppose (3.6) holds. Then the same argument applies if $q \leq 1$, and if $p \geq 1$, then the claim is a consequence of the uniqueness of the extension

$$
M_{\left(\omega_{1}\right)}^{\infty, q_{1}} \#_{A} M_{\left(\omega_{2}\right)}^{\infty, q_{2}} \subseteq M_{\left(\omega_{0}\right)}^{\infty, q},
$$

which is again proved in [7, Theorem 2.11]. Suppose $p<1<q$. If $q_{1}, q_{2} \geq 1$, then the uniqueness follows again from the uniqueness of (3.17). If $q_{1} \geq 1>q_{2}$, then it follows from the uniqueness of (3.17) with $q_{2}$ replaced by 1 , and analogously for $q_{2} \geq 1>q_{1}$. Finally if $q_{1}, q_{2}<1$, then the uniqueness follows from the uniqueness of (3.16).

Let $p, q \in(0, \infty]$ and set $r=\min (1, p, q)$. A particular case of Theorem 3.1 is the inclusion

$$
M_{\left(\omega_{0}\right)}^{p, q} \#_{A} M_{\left(\omega_{2}\right)}^{\infty, r} \subseteq M_{\left(\omega_{0}\right)}^{p, q},
$$


where the weights $\omega_{0}, \omega_{2} \in \mathscr{P}_{E}\left(\mathbf{R}^{4 d}\right)$ satisfy

$$
\omega_{0}\left(T_{A}(Z, X)\right) \lesssim \omega_{0}\left(T_{A}(Y, X)\right) \omega_{2}\left(T_{A}(Z, Y)\right), \quad X, Y, Z \in \mathbf{R}^{2 d},
$$

and $T_{A}$ is defined by (3.3).

We also note that $M_{(\omega)}^{p, q}$ is an algebra under the product $\#_{A}$ provided $p, q \in$ $(0, \infty], q \leq \min (1, p)$ and $\omega \in \mathscr{P}_{E}\left(\mathbf{R}^{4 d}\right)$ satisfies

$$
\omega\left(T_{A}(Z, X)\right) \lesssim \omega\left(T_{A}(Y, X)\right) \omega\left(T_{A}(Z, Y)\right), \quad X, Y, Z \in \mathbf{R}^{2 d} .
$$

\section{Necessary Conditions}

In this final section, we show that some of the sufficient conditions in Theorem 3.1 are necessary. We need the following lemma that concerns Wigner distributions.

Lemma 4.1. Let $q_{0}, q \in(0, \infty]$ satisfy $q_{0}<q$, let

$$
\phi(x)=\pi^{-\frac{d}{4}} e^{-\frac{1}{2}|x|^{2}} \quad \text { for } x \in \mathbf{R}^{d},
$$

let $\Lambda \subseteq \mathbf{R}^{d}$ be a lattice, let $\mathbf{c}=\{c(\kappa)\}_{\kappa \in \Lambda} \in \ell^{q}(\Lambda) \backslash \ell^{q_{0}}(\Lambda)$, where $c(\kappa) \geq 0$ for all $\kappa \in \Lambda$, and finally let

$$
f(x)=\sum_{\kappa \in \Lambda} c(\kappa) e^{i\langle x, \kappa\rangle} \phi(x) \in \mathscr{S}^{\prime}\left(\mathbf{R}^{d}\right) .
$$

Then

$$
f \in \bigcap_{p>0} M^{p, q}\left(\mathbf{R}^{d}\right) \backslash M^{\infty, q_{0}}\left(\mathbf{R}^{d}\right),
$$

and

$$
W_{f, \phi} \in \bigcap_{p>0} \mathcal{M}^{p, q}\left(\mathbf{R}^{2 d}\right)
$$

Proof. By replacing $\Lambda$ by a sufficiently dense lattice $\Lambda_{0}$, containing $\Lambda$ and letting $c(\kappa)=0$ when $\kappa \in \Lambda_{0} \backslash \Lambda$, we reduce ourselves to a situation where the hypothesis in Proposition 2.6 is fulfilled. Hence we may assume that (2.11) are dual frames for $L^{2}\left(\mathbf{R}^{d}\right)$.

First we show (4.1]) (cf. [28] Proposition 2.6]). On one hand, we have $\|f\|_{M^{p, q}} \lesssim$ $\|\mathbf{c}\|_{\ell^{q}}$ for any $p>0$ due to Proposition 2.6(1). Thus $f \in \bigcap_{p>0} M^{p, q}\left(\mathbf{R}^{d}\right)$. On the other hand $f \notin M^{\infty, q_{0}}\left(\mathbf{R}^{d}\right)$.

In fact, set $\phi_{1}(x)=(2 \pi)^{-\frac{d}{2}} e^{-\frac{1}{4}|x|^{2}}$ for $x \in \mathbf{R}^{d}$. Since

$$
V_{\phi} f(0, \iota)=(2 \pi)^{-\frac{d}{2}} \sum_{\kappa \in \Lambda} c(\iota-\kappa) e^{-\frac{1}{4}|\kappa|^{2}}=\mathbf{c} * \phi_{1}(\iota)
$$

we obtain

$$
\|\mathbf{c}\|_{\ell^{q_{0}}}^{q_{0}}=\sum_{\iota \in \Lambda} c(\iota)^{q_{0}} \leq(2 \pi)^{\frac{d q_{0}}{2}} \sum_{\iota \in \Lambda}\left(\mathbf{c} * \phi_{1}(\iota)\right)^{q_{0}}
$$


Y. Chen, J. Toft \& P. Wahlberg

$$
\begin{aligned}
& =(2 \pi)^{\frac{d q_{0}}{2}} \sum_{\iota \in \Lambda}\left|V_{\phi} f(0, \iota)\right|^{q_{0}} \leq(2 \pi)^{\frac{d q_{0}}{2}} \sum_{\iota \in \Lambda}\left(\sup _{j \in \Lambda}\left|V_{\phi} f(j, \iota)\right|\right)^{q_{0}} \\
& =(2 \pi)^{\frac{d q_{0}}{2}}\left\|V_{\phi} f\right\|_{\ell^{\infty, q_{0}}\left(\Lambda^{2}\right)}^{q_{0}} \asymp\|f\|_{M^{\infty}, q_{0}}^{q_{0}}
\end{aligned}
$$

again by Proposition 2.6. Thus it must hold $f \notin M^{\infty, q_{0}}\left(\mathbf{R}^{d}\right)$, since otherwise we get the contradiction $\mathbf{c} \in \ell^{q_{0}}(\Lambda)$. We have now showed (4.1).

In order to prove (4.2), set $a=W_{f, \phi} \in \mathscr{S}^{\prime}\left(\mathbf{R}^{2 d}\right)$. Since $\mathcal{M}^{p, q}$ is increasing with respect to $p$ and $q$, it suffices to intersect in (4.2) over $0<p \leq \min (1, q)$. We have

$$
\|a\|_{\mathcal{M}^{p, q}} \asymp\left\|V_{\Phi} W_{f, \phi}\right\|_{\ell^{p, q}\left(\Lambda^{4}\right)},
$$

where $\Phi(x, \xi)=(2 \pi)^{-\frac{d}{2}} e^{-\left(|x|^{2}+|\xi|^{2}\right)}$, and

$$
\Lambda^{4}=\Lambda \times \Lambda \times \Lambda \times \Lambda \subseteq \mathbf{R}^{4 d} .
$$

By straightforward computations, we get

$$
\begin{aligned}
a(x, \xi) & =W_{f, \phi}(x, \xi) \\
& =(2 \pi)^{-\frac{d}{2}} \sum_{\kappa \in \Lambda} c(\kappa) \pi^{-\frac{d}{2}} \int_{\mathbf{R}^{d}} e^{-\frac{1}{2}\left(\left|x-\frac{y}{2}\right|^{2}+\left|x+\frac{y}{2}\right|^{2}\right)} e^{i\left(\langle x, \kappa\rangle-\left\langle y, \xi-\frac{\kappa}{2}\right\rangle\right)} d y \\
& =2^{\frac{d}{2}} \pi^{-\frac{d}{2}} \sum_{\kappa \in \Lambda} c(\kappa) e^{-|x|^{2}-\left|\xi-\frac{\kappa}{2}\right|^{2}} e^{i\langle x, \kappa\rangle} .
\end{aligned}
$$

This gives

$$
V_{\Phi} a(x, \xi, \eta, y)=2^{\frac{d}{2}} \pi^{-\frac{d}{2}} \sum_{\kappa \in \Lambda} c(\kappa) F_{\kappa}(x, \xi, \eta, y)
$$

where

$$
\begin{aligned}
F_{\kappa}(x, \xi, \eta, y) & =(2 \pi)^{-\frac{3 d}{2}} \iint_{\mathbf{R}^{2 d}} e^{-\left(|z|^{2}+\left|\zeta-\frac{\kappa}{2}\right|^{2}+|z-x|^{2}-|\zeta-\xi|^{2}\right)} e^{-i(\langle z, \eta-\kappa\rangle+\langle y, \zeta\rangle)} d z d \zeta \\
& =2^{-\frac{5 d}{2}} \pi^{-\frac{d}{2}} e^{-\frac{1}{2}\left(|x|^{2}+\left|\xi-\frac{\kappa}{2}\right|^{2}\right)-\frac{1}{8}\left(|\eta-\kappa|^{2}+|y|^{2}\right)} e^{-\frac{i}{2}\left(\langle x, \eta-\kappa\rangle+\left\langle y, \xi+\frac{\kappa}{2}\right\rangle\right)}
\end{aligned}
$$

Hence

$$
\begin{aligned}
V_{\Phi} a(x, \xi, \eta, y)= & 2^{-2 d} \pi^{-d} \sum_{\kappa \in \Lambda} c(\kappa) e^{-\frac{1}{2}\left(|x|^{2}+\left|\xi-\frac{\kappa}{2}\right|^{2}\right)-\frac{1}{8}\left(|\eta-\kappa|^{2}+|y|^{2}\right)} \\
& \times e^{-\frac{i}{2}\left(\langle x, \eta-\kappa\rangle+\left\langle y, \xi+\frac{\kappa}{2}\right\rangle\right)}
\end{aligned}
$$

If $q<\infty$ we get, in the third inequality using $p \leq 1$,

$$
\begin{aligned}
\left\|W_{f, \phi}\right\|_{\mathcal{M}^{p, q}} & =\|a\|_{\mathcal{M}^{p, q}} \asymp\left\|\left\{V_{\Phi} a\left(k_{1}, \kappa_{1}, \kappa_{2}, k_{2}\right)\right\}_{k_{j}, \kappa_{j} \in \Lambda}\right\|_{\ell^{p, q}\left(\Lambda^{4}\right)} \\
& \lesssim\left(\sum_{k_{2}, \kappa_{2}}\left(\sum_{k_{1}, \kappa_{1}}\left(\sum_{\kappa} c(\kappa) e^{-\frac{1}{2}\left(\left|k_{1}\right|^{2}+\left|\kappa_{1}-\frac{\kappa}{2}\right|^{2}\right)-\frac{1}{8}\left(\left|\kappa_{2}-\kappa\right|^{2}+\left|k_{2}\right|^{2}\right)}\right)^{p}\right)^{\frac{q}{p}}\right)^{\frac{1}{q}}
\end{aligned}
$$




$$
\begin{aligned}
& \asymp\left(\sum_{\kappa_{2}}\left(\sum_{\kappa_{1}}\left(\sum_{\kappa} c(\kappa) e^{-\left(\frac{1}{2}\left|\kappa_{1}-\frac{\kappa}{2}\right|^{2}+\frac{1}{8}\left|\kappa_{2}-\kappa\right|^{2}\right)}\right)^{p}\right)^{\frac{q}{p}}\right)^{\frac{1}{q}} \\
& \leq\left(\sum_{\kappa_{2}}\left(\sum_{\kappa, \kappa_{1}} c(\kappa)^{p} e^{-\left(\frac{p}{2}\left|\kappa_{1}-\frac{\kappa}{2}\right|^{2}+\frac{p}{8}\left|\kappa_{2}-\kappa\right|^{2}\right)}\right)^{\frac{q}{p}}\right)^{\frac{1}{q}} \\
& \asymp\left(\sum_{\kappa_{2}}\left(\sum_{\kappa} c(\kappa)^{p} e^{-\frac{p}{8}\left|\kappa_{2}-\kappa\right|^{2}}\right)^{\frac{q}{p}}\right)^{\frac{1}{q}}=\left(\left\|\left\{c^{p}\right\} * e^{-\frac{p}{8}|\cdot|^{2}}\right\|_{\left.\ell^{\frac{q}{p}}\right)^{\frac{1}{p}}}\right. \\
& \leq\left(\left\|\left\{c^{p}\right\}\right\|_{\ell^{\frac{q}{p}}}\left\|e^{-\frac{p}{8}|\cdot|^{2}}\right\|_{\ell^{1}}\right)^{\frac{1}{p}} \asymp\|\mathbf{c}\|_{\ell^{q}}<\infty,
\end{aligned}
$$

using Young's inequality. The result follows if $q<\infty$. If $q=\infty$, a similar argument proves the result.

The preceding lemma is needed in the proof of Theorem 4.3 below on necessary conditions for continuity. We aim at conditions on the exponents $p_{j}, q_{j}, j=0,1,2$, that are necessary for

$$
\left\|a \#_{A} b\right\|_{M_{\left(\omega_{0}\right)}^{p_{0}, q_{0}}} \lesssim\|a\|_{M_{\left(\omega_{1}\right)}^{p_{1}, q_{1}}}\|b\|_{M_{\left(\omega_{2}\right)}^{p_{2}, q_{2}}}
$$

to hold for all $a, b \in \mathscr{S}\left(\mathbf{R}^{2 d}\right)$, for certain weight functions $\omega_{j}, j=0,1,2$. We restrict to weights of polynomial type.

By [36. Proposition 2.8], it suffices to prove the result in the Weyl case $A=1 / 2$, and then (4.4) in terms of symplectic modulation spaces is

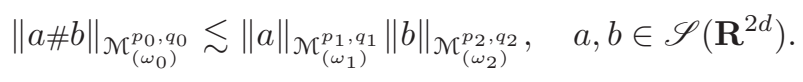

The conditions on the weights (3.2) and (3.3) are then transformed into

$$
\omega_{0}(Z+X, Z-X) \lesssim \omega_{1}(Y+X, Y-X) \omega_{2}(Z+Y, Z-Y), \quad X, Y, Z \in \mathbf{R}^{2 d} .
$$

(cf. 7], 23]).

We will consider weights with the particular structure

$$
\begin{aligned}
& \omega_{0}(X, Y)=\frac{\vartheta_{2}(X-Y)}{\vartheta_{0}(X+Y)}, \quad \omega_{1}(X, Y)=\frac{\vartheta_{2}(X-Y)}{\vartheta_{1}(X+Y)}, \\
& \omega_{2}(X, Y)=\frac{\vartheta_{1}(X-Y)}{\vartheta_{0}(X+Y)},
\end{aligned}
$$

for $\vartheta_{j} \in \mathscr{P}\left(\mathbf{R}^{2 d}\right), j=0,1,2$. Then (4.6) is automatically satisfied. Without loss, we may assume $\vartheta_{j} \in C^{\infty}[23$, Remark 2.18]. 
For $\vartheta \in \mathscr{P}\left(\mathbf{R}^{2 d}\right)$, let $S^{(\vartheta)}\left(\mathbf{R}^{2 d}\right)$ denote the space of smooth symbols on $\mathbf{R}^{2 d}$ such that $\left(\partial^{\alpha} a\right) / \vartheta \in L^{\infty}$ for any $\alpha \in \mathbf{N}^{2 d}$.

Lemma 4.2. Let $p, q \in(0, \infty]$, let $\vartheta_{j} \in \mathscr{P}\left(\mathbf{R}^{2 d}\right), j=1,2$ and suppose $\omega(X, Y)=$ $\vartheta_{2}(X-Y) / \vartheta_{1}(X+Y)$. Then there exist $a_{j} \in S^{\left(\vartheta_{j}\right)}\left(\mathbf{R}^{2 d}\right)$ and $b_{j} \in S^{\left(1 / \vartheta_{j}\right)}\left(\mathbf{R}^{2 d}\right)$, $j=1,2$ such that

$$
a_{j} \# b_{j}=b_{j} \# a_{j}=1, \quad j=1,2,
$$

and the map $a \mapsto a_{2} \# a \# b_{1}$ is continuous on $\mathscr{S}\left(\mathbf{R}^{2 d}\right)$ and extends uniquely to a homeomorphism from $\mathcal{M}_{(\omega)}^{p, q}\left(\mathbf{R}^{2 d}\right)$ to $\mathcal{M}^{p, q}\left(\mathbf{R}^{2 d}\right)$.

Proof. According to [2, Corollary 6.6] there exist $a_{j} \in S^{\left(\vartheta_{j}\right)}\left(\mathbf{R}^{2 d}\right)$ and $b_{j} \in$ $S^{\left(1 / \vartheta_{j}\right)}\left(\mathbf{R}^{2 d}\right), j=1,2$, such that (4.8) is satisfied.

By [23, Remark 2.18], we have

$$
S^{(\vartheta)}\left(\mathbf{R}^{2 d}\right)=\bigcap_{N \geq 0} \mathcal{M}_{\left(1 / \vartheta_{N}\right)}^{\infty, r}\left(\mathbf{R}^{2 d}\right), \quad \vartheta_{N}(X, Y)=\vartheta(X)\langle Y\rangle^{-N},
$$

for any $\vartheta \in \mathscr{P}\left(\mathbf{R}^{2 d}\right)$ and any $r>0$. More precisely the remark gives the equality for $r=1$, and for general $r>0$, the equality follows from the embeddings

$$
M_{\left(1 / v_{N}+N_{0}\right)}^{\infty, r_{2}} \subseteq M_{\left(1 / v_{N}\right)}^{\infty, r_{1}} \subseteq M_{\left(1 / v_{N}\right)}^{\infty, r_{2}}, \quad \text { when } \quad r_{1}<r_{2}, N_{0}>2 d\left(\frac{1}{r_{1}}-\frac{1}{r_{2}}\right) .
$$

If we set $r=\min (1, p, q)$, then $p_{1}=\infty, q_{1}=r, p_{2}=p, q_{2}=q$, as well as $p_{2}=\infty, q_{2}=r, p_{1}=p, q_{1}=q$, satisfy the conditions 3.4 and 3.5 or 3.6 of Theorem 3.1.

From these observations, the result follows from Theorem 3.1 and a repetition of the arguments in the proof of [7, Lemma 3.3].

Theorem 4.3. Let $p_{j}, q_{j} \in(0, \infty]$, suppose $\omega_{j} \in \mathscr{P}\left(\mathbf{R}^{4 d}\right), j=0,1,2$, are given by (4.7) where $\vartheta_{j} \in \mathscr{P}\left(\mathbf{R}^{2 d}\right), j=0,1,2$. If (4.5) holds then

$$
\frac{1}{p_{0}} \leq \frac{1}{p_{1}}+\frac{1}{p_{2}}, \quad \frac{1}{p_{0}} \leq \frac{1}{q_{1}}+\frac{1}{q_{2}} \quad \text { and } \quad q_{1}, q_{2} \leq q_{0}
$$

Proof. By Lemma 4.2, the estimate (4.5) with weights (4.7) implies

$$
\|a \# b\|_{\mathcal{M}^{p_{0}, q_{0}}} \lesssim\|a\|_{\mathcal{M}^{p_{1}, q_{1}}}\|b\|_{\mathcal{M}^{p_{2}, q_{2}}}, \quad a \in \mathcal{M}^{p_{1}, q_{1}}\left(\mathbf{R}^{2 d}\right), \quad b \in \mathscr{S}\left(\mathbf{R}^{2 d}\right) .
$$

It thus suffices to prove the result for $\omega_{j} \equiv 1, j=0,1,2$.

Let $a_{\lambda, \mu}(x, \xi)=e^{-\lambda|x|^{2}-\mu|\xi|^{2}}$ and $a_{\lambda}=a_{\lambda, \lambda}$, for $\mu, \lambda>0$. Then by the proof of [23. Proposition 3.1] (cf. 77, Sec. 3])

$$
\left\|a_{\lambda}\right\|_{\mathcal{M}^{p, q}}^{1 / d}=\pi^{\frac{1}{p}+\frac{1}{q}-1} p^{-\frac{1}{p}} q^{-\frac{1}{q}} \lambda^{-\frac{1}{p}}(1+\lambda)^{\frac{1}{p}+\frac{1}{q}-1}
$$

and

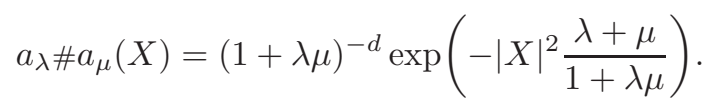


Hence

$$
\left\|a_{\lambda} \# a_{\lambda}\right\|_{\mathcal{M}^{p, q}}^{1 / d}=\pi^{1 / p+1 / q-1} p^{-1 / p} q^{-1 / q}\left(1+\lambda^{2}\right)^{-1 / q}(2 \lambda)^{-1 / p}(1+\lambda)^{2(1 / p+1 / q-1)} .
$$

Thus

$$
\begin{aligned}
\left(\frac{\left\|a_{\lambda} \# a_{\lambda}\right\| \mathcal{\mathcal { N }}_{p_{0}, q_{0}}}{\left\|a_{\lambda}\right\|_{\mathcal{M}^{p_{1}, q_{1}}}\left\|a_{\lambda}\right\|_{\mathcal{M}^{p_{2}, q_{2}}}}\right)^{1 / d}= & C \lambda^{\frac{1}{p_{1}}+\frac{1}{p_{2}}-\frac{1}{p_{0}}} \\
& \times\left(1+\lambda^{2}\right)^{-\frac{1}{q_{0}}}(1+\lambda)^{\frac{2}{p_{0}}-\frac{1}{p_{1}}-\frac{1}{p_{2}}+\frac{2}{q_{0}}-\frac{1}{q_{1}}-\frac{1}{q_{2}}},
\end{aligned}
$$

for some constant $C>0$ which does not depend on $\lambda$. The right hand side behaves like $\lambda^{\frac{1}{p_{0}}-\frac{1}{q_{1}}-\frac{1}{q_{2}}}$ when $\lambda$ is large, and like $\lambda^{\frac{1}{p_{1}}+\frac{1}{p_{2}}-\frac{1}{p_{0}}}$ when $\lambda$ is small. The continuity (4.10) hence implies the necessary conditions

$$
\frac{1}{p_{0}} \leq \frac{1}{q_{1}}+\frac{1}{q_{2}}, \quad \frac{1}{p_{0}} \leq \frac{1}{p_{1}}+\frac{1}{p_{2}} .
$$

It remains to show $q_{1}, q_{2} \leq q_{0}$. Since $\overline{a_{1} \# a_{2}}=\bar{a}_{2} \# \bar{a}_{1}$ (cf. 23]), it suffices to show $q_{1} \leq q_{0}$. We give a proof by contradiction. Suppose (4.10) holds and $q_{0}<q_{1}$. Let $\Lambda \subseteq \mathbf{R}^{d}$ be a lattice,

$$
\phi(x)=\pi^{-\frac{d}{4}} e^{-\frac{1}{2}|x|^{2}}, \quad \mathbf{c}=\{c(\kappa)\}_{\kappa \in \Lambda} \in \ell^{q_{1}}(\Lambda) \backslash \ell^{q_{0}}(\Lambda),
$$

and let

$$
f(x)=\sum_{\kappa \in \Lambda} c(\kappa) e^{i\langle x, \kappa\rangle} \phi(x) .
$$

Then

$$
\begin{aligned}
& f \in \bigcap_{p_{1}>0} M^{p_{1}, q_{1}}\left(\mathbf{R}^{d}\right) \backslash M^{\infty, q_{0}}\left(\mathbf{R}^{d}\right), \\
& a=W_{f, \phi} \in \bigcap_{p_{1}>0} \mathcal{M}^{p_{1}, q_{1}}\left(\mathbf{R}^{2 d}\right) \text { and } b=W_{\phi, \phi} \in \mathscr{S}\left(\mathbf{R}^{2 d}\right),
\end{aligned}
$$

by Lemma 4.1. Since

$$
\mathrm{Op}^{w}(a) g=(2 \pi)^{-\frac{d}{2}}(g, \phi) f \quad \text { and } \quad \mathrm{Op}^{w}(b) g=(2 \pi)^{-\frac{d}{2}}(g, \phi) \phi,
$$

it follows that

$$
\mathrm{Op}^{w}(a \# b) \phi=(2 \pi)^{-d}\|\phi\|_{L^{2}}^{4} f \in \bigcap_{p_{1}>0} M^{p_{1}, q_{1}}\left(\mathbf{R}^{d}\right) \backslash M^{\infty, q_{0}}\left(\mathbf{R}^{d}\right) .
$$

Therefore $\mathrm{Op}^{w}(a \# b)$ is not continuous from $\mathscr{S}\left(\mathbf{R}^{d}\right)$ to $M^{\infty, q_{0}}\left(\mathbf{R}^{d}\right)$.

On the other hand, we have by assumption

$$
a \# b \in \mathcal{M}^{p_{0}, q_{0}} \subseteq \mathcal{M}^{\infty, q_{0}} .
$$

If $q_{0} \in(0,1]$, then $\mathrm{Op}^{w}(a \# b)$ is continuous from $M^{p_{0}, q_{0}}$ to $M^{p_{0}, q_{0}}$ when $p_{0} \in\left[q_{0}, \infty\right]$, by [35. Theorem 3.1]. This contradicts the fact that $\mathrm{Op}^{w}(a \# b)$ is not continuous from $\mathscr{S}$ to $M^{\infty, q_{0}}$. Hence the assumption $q_{0}<q_{1}$ must be false. 
If instead $q_{0} \in[1, \infty]$, then by [31, Theorem 4.3] $\mathrm{Op}^{w}(a \# b)$ is continuous from $M^{1,1}$ to $M^{q_{0}, q_{0}}$, which again contradicts the fact that $\mathrm{Op}^{w}(a \# b)$ is not continuous from $\mathscr{S}$ to $M^{\infty, q_{0}}$. Hence the assumption $q_{0}<q_{1}$ is again false.

Thus we must have $q_{1} \leq q_{0}$.

Remark 4.4. Let $\mathscr{P}_{E}^{0}\left(\mathbf{R}^{d}\right)$ denote all $\omega \in \mathscr{P}_{E}\left(\mathbf{R}^{d}\right)$ such that $\omega$ is $v$-moderate for a sub-multiplicative weight $v$ satisfying

$$
v(x) \lesssim e^{r|x|}, \quad x \in \mathbf{R}^{d}
$$

for all $r>0$. Then $\mathscr{P}\left(\mathbf{R}^{d}\right) \subsetneq \mathscr{P}_{E}^{0}\left(\mathbf{R}^{d}\right)$. By using the new [1, Theorem 4.1] instead of Lemma 4.2 it follows that Theorem 4.3 holds for $\vartheta_{j} \in \mathscr{P}_{E}^{0}\left(\mathbf{R}^{2 d}\right)$ and $\omega_{j}$ defined by (4.7). The space $\mathscr{S}$ in (4.5) is then replaced by $\mathcal{S}_{1}$.

Remark 4.5. For Banach modulation spaces with exponents $p_{j}, q_{j}$ restricted to $[1, \infty]$, we have found that the following conditions are necessary and sufficient for continuity of the Weyl product [7, Theorems 0.1 and 3.1].

$$
\begin{aligned}
\frac{1}{p_{0}} & \leq \frac{1}{p_{1}}+\frac{1}{p_{2}} \\
q_{1}, q_{2} & \leq q_{0}, \quad 1 \leq \frac{1}{q_{1}}+\frac{1}{q_{2}} \\
\frac{1}{p_{0}}+\frac{1}{q_{0}} & \leq \frac{1}{q_{1}}+\frac{1}{q_{2}}, \quad 1+\frac{1}{q_{0}} \leq \frac{1}{q_{1}}+\frac{1}{q_{2}}+\frac{1}{p_{j}}, \quad j=1,2, \\
1+\frac{1}{p_{0}}+\frac{1}{q_{0}} & \leq \frac{1}{q_{1}}+\frac{1}{q_{2}}+\frac{1}{p_{1}}+\frac{1}{p_{2}} .
\end{aligned}
$$

In this paper, we have worked with exponents $p_{j}, q_{j}$ in the full range $(0, \infty]$. The sufficient conditions in Theorem 3.1 and the necessary conditions in Theorem 4.3 are not equal, as conditions (4.11)-4.14 are for exponents in $[1, \infty]$.

In fact, consider the inclusion

$$
M^{1,2} \# M^{1,2} \subseteq M^{\infty, 2},
$$

which holds since the exponents satisfy (4.11)-(4.14). They do however not satisfy (3.4), and (3.5) or (3.6). Hence the sufficient conditions in Theorem 3.1 are not at all necessary.

\section{Appendix}

In this appendix, we prove the formula

$$
\mathscr{F}(f \bar{\phi})(\xi)=(2 \pi)^{-\frac{d}{2}}\left(f, \phi e^{i\langle\cdot, \xi\rangle}\right), \quad f \in \mathcal{S}_{s}^{\prime}\left(\mathbf{R}^{d}\right), \quad \phi \in \mathcal{S}_{s}\left(\mathbf{R}^{d}\right), \quad \xi \in \mathbf{R}^{d},
$$

for $s \geq \frac{1}{2}$, which we claimed to be true in the definition of the STFT (2.5). There is a parallel formula for $f \in \Sigma_{s}^{\prime}\left(\mathbf{R}^{d}\right), \phi \in \Sigma_{s}\left(\mathbf{R}^{d}\right)$ and $s>\frac{1}{2}$, that we also prove. 
Let $f \in \mathcal{S}_{s}^{\prime}\left(\mathbf{R}^{d}\right)$, let $\phi \in \mathcal{S}_{s}\left(\mathbf{R}^{d}\right)$ and denote

$$
u(\xi)=(2 \pi)^{-\frac{d}{2}}\left(f, \phi e^{i\langle\cdot, \xi\rangle}\right)=(2 \pi)^{-\frac{d}{2}}\left\langle f, \bar{\phi} e^{-i\langle\cdot, \xi\rangle}\right\rangle, \quad \xi \in \mathbf{R}^{d} .
$$

Then $u \in C^{\infty}\left(\mathbf{R}^{d}\right)$. We need the following estimate (cf. [5]).

Lemma A.1. The function (A.2) satisfies the estimate

$$
|u(\xi)| \lesssim e^{c|\xi|^{\frac{1}{s}}}, \quad \xi \in \mathbf{R}^{d}
$$

for any $c>0$.

Proof. By 2.4 $\phi \in \mathcal{S}_{s, h}\left(\mathbf{R}^{d}\right)$ for all $h \geq h_{0}$ where $h_{0}>0$. Let $c>0$ and set

$$
h=\max \left(h_{0},\left(\frac{d s}{c}\right)^{s}\right) .
$$

Let $\alpha, \beta \in \mathbf{N}^{d}$. Using $|\gamma| ! \leq d^{|\gamma|} \gamma$ ! (cf. [27 Eq. (0.3.3)]) and $\sum_{\gamma \leq \beta}\left(\begin{array}{c}\beta \\ \gamma\end{array}\right)=2^{|\beta|}$ we estimate for $x, \xi \in \mathbf{R}^{d}$

$$
\begin{aligned}
& \frac{\left|x^{\alpha} \partial_{x}^{\beta}\left(e^{i\langle x, \xi\rangle} \phi(x)\right)\right|}{(\alpha ! \beta !)^{s}(2 h)^{|\alpha+\beta|}} \leq 2^{-|\alpha+\beta|} \sum_{\gamma \leq \beta}\left(\begin{array}{c}
\beta \\
\gamma
\end{array}\right) \frac{|\xi|^{|\gamma|} h^{-|\gamma|}}{\gamma !^{s}} \frac{\left|x^{\alpha} \partial^{\beta-\gamma} \phi(x)\right|}{(\alpha !(\beta-\gamma) !)^{s} h^{|\alpha+\beta-\gamma|}}\left(\begin{array}{c}
\beta \\
\gamma
\end{array}\right)^{-s} \\
& \leq\|\phi\|_{\mathcal{S}_{s, h}} 2^{-|\alpha+\beta|} \sum_{\gamma \leq \beta}\left(\begin{array}{l}
\beta \\
\gamma
\end{array}\right)\left(\frac{\left(\frac{c}{s}|\xi|^{\frac{1}{s}}\right)^{|\gamma|}}{|\gamma| !}\right)^{s}\left(\frac{(d s)^{s}}{h c^{s}}\right)^{|\gamma|} \\
& \lesssim 2^{-|\alpha+\beta|} \sum_{\gamma \leq \beta}\left(\begin{array}{l}
\beta \\
\gamma
\end{array}\right)\left(\frac{\left(\frac{c}{s}|\xi|^{\frac{1}{s}}\right)^{|\gamma|}}{|\gamma| !}\right)^{s} \\
& \leq e^{c|\xi|^{\frac{1}{s}}} 2^{-|\alpha+\beta|} \sum_{\gamma \leq \beta}\left(\begin{array}{l}
\beta \\
\gamma
\end{array}\right) \\
& \leq e^{c|\xi|^{\frac{1}{s}}}
\end{aligned}
$$

This implies

$$
\left\|\phi e^{i\langle\cdot, \xi\rangle}\right\|_{\mathcal{S}_{s, 2 h}} \lesssim e^{c|\xi|^{\frac{1}{s}}}, \quad \xi \in \mathbf{R}^{d},
$$

which via (2.4a) finally gives the estimate

$$
|u(\xi)| \lesssim\left\|\phi e^{i\langle\cdot, \xi\rangle}\right\|_{\mathcal{S}_{s, 2 h}} \lesssim e^{c|\xi|^{\frac{1}{s}}}, \quad \xi \in \mathbf{R}^{d} .
$$

The formula (A.1) amounts to the claim $\mathscr{F}(f \bar{\phi})=u$.

A priori $\mathscr{F}(f \bar{\phi}) \in \mathcal{S}_{s}^{\prime}\left(\mathbf{R}^{d}\right)$ is the distribution

$$
\langle\mathscr{F}(f \bar{\phi}), g\rangle=\langle f, \bar{\phi} \widehat{g}\rangle, \quad g \in \mathcal{S}_{s}\left(\mathbf{R}^{d}\right) .
$$

To prove our claim $\mathscr{F}(f \bar{\phi})=u$ we must therefore show

$$
\langle f, \bar{\phi} \widehat{g}\rangle=\int_{\mathbf{R}^{d}} u(x) g(x) d x=(2 \pi)^{-d / 2} \int_{\mathbf{R}^{d}}\left\langle f, \bar{\phi} g(x) e^{-i\langle\cdot, x\rangle}\right\rangle d x, \quad g \in \mathcal{S}_{s}\left(\mathbf{R}^{d}\right) .
$$


Note that the integral is well defined due to Lemma A.1 and the estimate for $g \in \mathcal{S}_{s}\left(\mathbf{R}^{d}\right)$ 33, Lemma 1.6]

$$
|g(x)| \lesssim e^{-\varepsilon|x|^{1 / s}}, \quad x \in \mathbf{R}^{d}
$$

which is valid for some $\varepsilon>0$.

In view of the definition of the Fourier transform $\widehat{g}$, formula (A.3) is true provided we can switch order in the action of the distribution $1 \otimes f \in \mathcal{S}_{s}^{\prime}\left(\mathbf{R}^{2 d}\right)$ with respect to the first and second $\mathbf{R}^{d}$ variable, when it acts on the test function $\Phi(x, y)=(2 \pi)^{-d / 2} \bar{\phi}(y) g(x) e^{-i\langle y, x\rangle}$. Note that $\Phi \in \mathcal{S}_{s}\left(\mathbf{R}^{2 d}\right)$ if $\phi, g \in \mathcal{S}_{s}\left(\mathbf{R}^{d}\right)$ and $s \geq \frac{1}{2}$, and $\Phi \in \Sigma_{s}\left(\mathbf{R}^{2 d}\right)$ if $\phi, g \in \Sigma_{s}\left(\mathbf{R}^{d}\right)$ and $s>\frac{1}{2}$, cf. [3. Theorem 3.1] and 4, Proposition 3.4].

Thus the claim (A.1) is a consequence of the following Fubini-type result for Gelfand-Shilov distributions. It corresponds to [22] Theorem 5.1.1] in the Schwartz distribution theory.

Theorem A.2. Suppose $s \geq \frac{1}{2}$, and $f_{j} \in \mathcal{S}_{s}^{\prime}\left(\mathbf{R}^{d_{j}}\right), j=1,2$. Then there exists a unique tensor product distribution $f=f_{1} \otimes f_{2} \in \mathcal{S}_{s}^{\prime}\left(\mathbf{R}^{d_{1}+d_{2}}\right)$ such that

$$
\left\langle f_{1} \otimes f_{2}, \phi_{1} \otimes \phi_{2}\right\rangle=\left\langle f_{1}, \phi_{1}\right\rangle\left\langle f_{2}, \phi_{2}\right\rangle, \quad \phi_{j} \in \mathcal{S}_{s}\left(\mathbf{R}^{d_{j}}\right), \quad j=1,2 .
$$

It holds

$$
\langle f, \phi\rangle=\left\langle f_{1},\left\langle f_{2}, \phi\left(x_{1}, x_{2}\right)\right\rangle\right\rangle=\left\langle f_{2},\left\langle f_{1}, \phi\left(x_{1}, x_{2}\right)\right\rangle\right\rangle, \quad \phi \in \mathcal{S}_{s}\left(\mathbf{R}^{d_{1}+d_{2}}\right),
$$

where $f_{j}$ acts on $x_{j}$ only, $j=1,2$.

The same conclusion holds for $s>1 / 2$ and $f_{j} \in \Sigma_{s}^{\prime}\left(\mathbf{R}^{d_{j}}\right), j=1,2$, with test functions in $\Sigma_{s}$.

Proof. We use the Hermite functions

$$
h_{\alpha}(x)=\pi^{-\frac{d}{4}}(-1)^{|\alpha|}\left(2^{|\alpha|} \alpha !\right)^{-\frac{1}{2}} e^{\frac{|x|^{2}}{2}}\left(\partial^{\alpha} e^{-|x|^{2}}\right), \quad x \in \mathbf{R}^{d}, \quad \alpha \in \mathbf{N}^{d},
$$

and formal series expansions with respect to Hermite functions:

$$
f=\sum_{\alpha \in \mathbf{N}^{d}} c_{\alpha} h_{\alpha},
$$

where $\left\{c_{\alpha}\right\}$ is a sequence of complex coefficients defined by $c_{\alpha}=c_{\alpha}(f)=\left(f, h_{\alpha}\right)$.

It is known that Gelfand-Shilov spaces and their distribution duals can be identified by means of such series expansions, with characterizations in terms of the corresponding sequence spaces (see [14, 15, 37] and the references therein).

In fact, let

$$
f=\sum_{\alpha \in \mathbf{N}^{d}} c_{\alpha} h_{\alpha},
$$


and

$$
\phi=\sum_{\alpha \in \mathbf{N}^{d}} d_{\alpha} h_{\alpha}
$$

with sequences $\left\{c_{\alpha}\right\}$ and $\left\{d_{\alpha}\right\}$ of finite support. Then the sesquilinear form

$$
(f, \phi)=\sum_{\alpha \in \mathbf{N}^{d}} c_{\alpha} \overline{d_{\alpha}},
$$

agrees with the inner product on $L^{2}\left(\mathbf{R}^{d}\right)$ due to the fact that $\left\{h_{\alpha}\right\}_{\alpha \in \mathbf{N}^{d}} \subseteq L^{2}\left(\mathbf{R}^{d}\right)$ is an orthonormal basis. The form (A.4) extends uniquely to the duality on $\mathcal{S}_{s}^{\prime}\left(\mathbf{R}^{d}\right) \times$ $\mathcal{S}_{s}\left(\mathbf{R}^{d}\right)$ for $s \geq \frac{1}{2}$, and to the duality on $\Sigma_{s}^{\prime}\left(\mathbf{R}^{d}\right) \times \Sigma_{s}\left(\mathbf{R}^{d}\right)$ for $s>\frac{1}{2}$. All spaces are then expressed in terms of the Hilbert sequence spaces

$$
\ell_{r}^{2}=\ell_{r}^{2}\left(\mathbf{N}^{d}\right)=\left\{\left\{c_{\alpha}\right\} ; \sum_{\alpha \in \mathbf{N}^{d}}\left|c_{\alpha}\right|^{2} e^{r|\alpha|^{\frac{1}{2 s}}}<\infty\right\}
$$

where $r \in \mathbf{R}$. For $s \geq \frac{1}{2}$ the space $\mathcal{S}_{s}\left(\mathbf{R}^{d}\right)$ is identified topologically as the inductive limit

$$
\mathcal{S}_{s}\left(\mathbf{R}^{d}\right)=\bigcup_{r>0}\left\{\sum_{\alpha \in \mathbf{N}^{d}} c_{\alpha} h_{\alpha} ;\left\{c_{\alpha}\right\} \in \ell_{r}^{2}\right\},
$$

and $\mathcal{S}_{s}^{\prime}\left(\mathbf{R}^{d}\right)$ is identified topologically as the projective limit

$$
\mathcal{S}_{s}^{\prime}\left(\mathbf{R}^{d}\right)=\bigcap_{r>0}\left\{\sum_{\alpha \in \mathbf{N}^{d}} c_{\alpha} h_{\alpha} ;\left\{c_{\alpha}\right\} \in \ell_{-r}^{2}\right\} .
$$

For $s>\frac{1}{2}$, the space $\Sigma_{s}\left(\mathbf{R}^{d}\right)$ is identified topologically as the projective limit

$$
\Sigma_{s}\left(\mathbf{R}^{d}\right)=\bigcap_{r>0}\left\{\sum_{\alpha \in \mathbf{N}^{d}} c_{\alpha} h_{\alpha}:\left\{c_{\alpha}\right\} \in \ell_{r}^{2}\right\},
$$

and $\Sigma_{s}^{\prime}\left(\mathbf{R}^{d}\right)$ is identified topologically as the inductive limit

$$
\Sigma_{s}^{\prime}\left(\mathbf{R}^{d}\right)=\bigcup_{r>0}\left\{\sum_{\alpha \in \mathbf{N}^{d}} c_{\alpha} h_{\alpha} ;\left\{c_{\alpha}\right\} \in \ell_{-r}^{2}\right\} .
$$

We have for $\alpha=\left(\alpha_{1}, \alpha_{2}\right) \in \mathbf{N}^{d_{1}+d_{2}}$ with $\alpha_{j} \in \mathbf{N}^{d_{j}}, j=1,2, h_{\alpha}=h_{\alpha_{1}} \otimes h_{\alpha_{2}}$. This gives for $f_{j} \in \mathcal{S}_{s}^{\prime}\left(\mathbf{R}^{d_{j}}\right), j=1,2$,

$$
c_{\alpha}=c_{\alpha}\left(f_{1} \otimes f_{2}\right)=\left(f_{1}, h_{\alpha_{1}}\right)\left(f_{2}, h_{\alpha_{2}}\right), \quad \alpha=\left(\alpha_{1}, \alpha_{2}\right) \in \mathbf{N}^{d_{1}+d_{2}},
$$

so $c_{\alpha}=c_{1, \alpha_{1}} c_{2, \alpha_{2}}$ if we denote $c_{j, \alpha_{j}}=\left(f_{j}, h_{\alpha_{j}}\right)$ where $\alpha_{j} \in \mathbf{N}^{d_{j}}$ for $j=1,2$. 
Let $\phi \in \mathcal{S}_{s}\left(\mathbf{R}^{d_{1}+d_{2}}\right)$ and denote $d_{\alpha}(\phi)=\left(\phi, h_{\alpha}\right)$ for $\alpha \in \mathbf{N}^{d_{1}+d_{2}}$. This gives for any $r>0$

$$
\left\langle f_{1} \otimes f_{2}, \phi\right\rangle=\sum_{\left(\alpha_{1}, \alpha_{2}\right) \in \mathbf{N}^{d_{1}+d_{2}}} c_{1, \alpha_{1}} c_{2, \alpha_{2}} e^{-r\left|\left(\alpha_{1}, \alpha_{2}\right)\right| \frac{1}{2 s}} \overline{d_{\alpha_{1}, \alpha_{2}}} e^{r\left|\left(\alpha_{1}, \alpha_{2}\right)\right|^{\frac{1}{2 s}}} .
$$

From

$$
e^{-r\left|\left(\alpha_{1}, \alpha_{2}\right)\right|^{\frac{1}{2 s}}} \leq e^{-\frac{r}{2}\left|\alpha_{1}\right|^{\frac{1}{2 s}}} e^{-\frac{r}{2}\left|\alpha_{2}\right|^{\frac{1}{2 s}}}
$$

$\left\{c_{1, \alpha_{1}}\right\} \in \ell_{-r}^{2}\left(\mathbf{N}^{d_{1}}\right),\left\{c_{2, \alpha_{2}}\right\} \in \ell_{-r}^{2}\left(\mathbf{N}^{d_{2}}\right)$ for any $r>0,\left\{d_{\alpha_{1}, \alpha_{2}}\right\} \in \ell_{r}^{2}\left(\mathbf{N}^{d_{1}+d_{2}}\right)$ for some $r>0$, and the Cauchy-Schwarz inequality, we may now conclude that the sum (A.5) converges absolutely.

The conclusion of the theorem is thus a consequence of the well-known Fubini theorem with respect to the counting measure.

\section{References}

[1] A. Abdeljawad, S. Coriasco and J. Toft, Liftings for Ultra-Modulation Spaces, and One-Parameter Groups of Gevrey Type Pseudo-Differential Operators, arXiv:1712.04338.

[2] J. M. Bony and J. Y. Chemin, Espaces fonctionnels associés au calcul de WeylHörmander, Bull. Soc. Math. France 122 (1994) 77-118.

[3] M. Cappiello and J. Toft, Pseudo-differential operators in a Gelfand-Shilov setting, Math. Nachr. 290 (2017) 738-755.

[4] E. Carypis and P. Wahlberg, Propagation of exponential phase space singularities for Schrödinger equations with quadratic Hamiltonians, J. Fourier Anal. Appl. 23 (2017) 530-571.

[5] J. Chung, S.-Y. Chung and D. Kim, Characterizations of the Gelfand-Shilov spaces via Fourier transforms, Proc. Amer. Math. Soc. 124 (1996) 2101-2108.

[6] E. Cordero, S. Pilipović, L. Rodino and N. Teofanov, Quasianalytic GelfandShilov spaces with applications to localization operators, Rocky Mountain J. Math. 40 (2010) 1123-1147.

[7] E. Cordero, J. Toft and P. Wahlberg, Sharp results for the Weyl product on modulation spaces, J. Funct. Anal. 267 (2014) 3016-3057.

[8] H. G. Feichtinger, Modulation Spaces on Locally Compact Abelian Groups. Technical Report, University of Vienna, Vienna, 1983, M. Krishna, R. Radha and S. Thangavelu, Wavelets and Their Applications (Allied Publishers Private Limited, New Delhi, Mumbai, Kolkata, Chennai, Nagpur, Ahmedabad, Bangalore, Hyderabad, Lucknow, 2003), pp. 99-140.

[9] H. G. Feichtinger and K. Gröchenig, Banach spaces related to integrable group representations and their atomic decompositions, I, J. Funct. Anal. 86 (1989) 307-340.

[10] H. G. Feichtinger and K. Gröchenig, Banach spaces related to integrable group representations and their atomic decompositions, II, Monatsh. Math. 108 (1989) 129-148.

[11] Y. V. Galperin and S. Samarah, Time-frequency analysis on modulation spaces $M_{m}^{p, q}$, $0<p, q \leq \infty$, Appl. Comput. Harmon. Anal. 16 (2004) 1-18.

[12] I. M. Gel'fand and G. E. Shilov, Generalized Functions, I-III (Academic Press, New York London, 1968). 
[13] T. Gramchev, S. Pilipović and L. Rodino, Classes of Degenerate Elliptic Operators in Gelfand-Shilov Spaces, in New Developments in Pseudo-Differential Operators, Operator Theory: Advances and Applications, eds. L. Rodino and M. W. Wong, Vol. 189 (Birkhäuser Verlag, Basel, 2009), pp. 15-31.

[14] T. Gramchev, S. Pilipović and L. Rodino, Eigenfunction expansions in $\mathbb{R}^{n}$, Proc. Amer. Math. Soc. 139 (2011) 4361-4368.

[15] T. Gramchev, A. Lecke, S. Pilipović and L. Rodino, Gelfand-Shilov Type Spaces Through Hermite Expansions, in Pseudo-Differential Operators and Generalized Functions, Operator Theory: Advances and Applications, eds. S. Pilipović and J. Toft (Birkhäuser, Basel Heidelberg, New York, Dordrecht, London, 2015), pp. 95-105.

[16] K. Gröchenig, Describing functions: Atomic decompositions versus frames, Monatsh. Math. 112 (1991) 1-42.

[17] K. Gröchenig, Foundations of Time-Frequency Analysis (Birkhäuser, Boston, 2001).

[18] K. Gröchenig, Composition and spectral invariance of pseudodifferential operators on modulation spaces, J. Anal. Math. 98 (2006) 65-82.

[19] K. Gröchenig, Weight functions in time-frequency analysis, in Pseudodifferential Operators: Partial Differential Equations and Time-Frequency Analysis, Fields Institute Communication, Vol. 52, eds. L. Rodino and M. Wang (American Mathematical Society, 2007), pp. 343-366.

[20] K. Gröchenig and Z. Rzeszotnik, Banach algebras of pseudodifferential operators and their almost diagonalization, Ann. Inst. Fourier 58(7) (2008) 2279-2314.

[21] K. Gröchenig and G. Zimmermann, Spaces of test functions via the STFT, J. Funct. Spaces Appl. 2 (2004) 25-53.

[22] L. Hörmander, The Analysis of Linear Partial Differential Operators, Vols. I-III (Springer-Verlag, Berlin Heidelberg, NewYork, Tokyo, 1983, 1985).

[23] A. Holst, J. Toft and P. Wahlberg, Weyl product algebras and modulation spaces, J. Funct. Anal. 251 (2007) 463-491.

[24] A. J. E. M. Janssen and S. J. L. Eijndhoven, Spaces of type W, growth of Hermite coefficients, Wigner distribution, and Bargmann transform, J. Math. Anal. Appl. 152 (1990) 368-390.

[25] S. Kostadinova, K. Saneva and J. Vindas, Gabor frames and asymptotic behavior of Schwartz distributions, Appl. Anal. Discrete Math. 10 (2016) 292-307.

[26] D. Labate, Time-frequency analysis of pseudodifferential operators, Monatshefte Math. 133 (2001) 143-156.

[27] F. Nicola and L. Rodino, Global Pseudo-Differential Calculus on Euclidean Spaces (Birkhäuser, 2010).

[28] M. Reich, A non-analytic superposition result on Gevrey-modulation spaces, arXiv:1411.3206.

[29] J. Sjöstrand, An algebra of pseudodifferential operators, Math. Res. L. 1 (1994) 185192.

[30] J. Toft, Subalgebras to a Wiener type algebra of pseudo-differential operators, Ann. Inst. Fourier 51 (2001) 1347-1383.

[31] J. Toft, Continuity properties for modulation spaces with applications to pseudodifferential calculus, I, J. Funct. Anal. 207 (2004) 399-429.

[32] J. Toft, Continuity and Schatten properties for pseudo-differential operators on modulation spaces, in Modern Trends in Pseudo-Differential Operators, Operator 
Theory: Advances and Applications, Vol. 172, eds. J. Toft, M. W. Wong and H. Zhu (Birkhäuser Verlag, Basel, 2007), pp. 173-206.

[33] J. Toft, The Bargmann transform on modulation and Gelfand-Shilov spaces, with applications to Toeplitz and pseudo-differential operators, J. Pseudo-Differ. Oper. Appl. 3 (2012) 145-227.

[34] J. Toft, Gabor analysis for a broad class of quasi-Banach modulation spaces, in Pseudo-Differential Operators and Generalized Functions, Operator Theory: Advances and Applications, Vol. 245, eds. S. Pilipović and J. Toft (Birkhäuser, Basel Heidelberg, New York, Dordrecht, London, 2015), pp. 249-278.

[35] J. Toft, Continuity and compactness for pseudo-differential operators with symbols in quasi-Banach spaces or Hörmander classes, Anal. Appl. 15 (2017) 353-389.

[36] J. Toft, Matrix parameterized pseudo-differential calculi on modulation spaces, in Generalized Functions and Fourier Analysis, Operator Theory: Advances and Applications, Vol. 260, eds. M. Oberguggenberger, J. Toft, J. Vindas and P. Wahlberg (Birkhäuser, 2017), pp. 215-235.

[37] J. Toft, Images of function and distribution spaces under the Bargmann transform, J. Pseudo-Differ. Oper. Appl. 8 (2017) 83-139.

[38] P. Wahlberg and P. J. Schreier, Gabor discretization of the Weyl product for modulation spaces and filtering of nonstationary stochastic processes, Appl. Comput. Harmon. Anal. 26 (2009) 97-120. 